\title{
Multiple Politico-Economic Regimes, Inequality and Growth
}

\author{
Alain Desdoigts* and Fabien Moizeau**
}

May 2001

\begin{abstract}
In this paper, we abandon the stylized median voter and study (i) how distributional tensions can act in many subtle ways depending on social affinity and on the prospect of upward or downward mobility of the different income groups, (ii) both the income distribution and growth dynamics. In a world in which both neighborhood and family attributes are important to determine the investment effort and the productivity of education and therefore social mobility, we find multiple politico-economic regimes that are supported by new international empirical evidence. In particular, we highlight an alternative mechanism through which the pressure for redistribution can be highly non-linear therefore providing an explanation as to why more equal and integrated economies may redistribute more, not less. Our framework displays multiple steady states which depend on historical economic discrimination, but an economy's institutions will have to overcome ranges of inequality in which voting cycles are likely to emerge along the transitional path.
\end{abstract}

Keywords: Clubs formation, growth, human capital, redistribution, and social mobility.

JEL Classification: D31, H2, I22, J62, P16.

\footnotetext{
0 We thank, without implications, Micaël Castanheira, Hervé Crès, Mathias Dewatripont, Fernando Jaramillo, Gérard Roland, Stéphane Rossignol, Bertrand Wigniolle, and seminar participants at ECARES (Brussels), EPEE (Evry), EUREQua (Paris 1), the 6th Coalition Formation Workshop (CORE, Louvain-la-Neuve), and the 2001 Annual Meeting of the European Public Choice Society (Paris). This research is partially supported by the Centre National de la Recherche Scientifique, and the Direction de la Recherche, des Etudes, de l'Evaluation et des Statistiques - Mission Recherche du Ministère de l'emploi et de la solidarité. It was carried out in part in the Sonderforschungsbereich 373 at Humboldt-Universität zu Berlin whose hospitality is acknowledged. The usual disclaimers apply.

* Correspondence to: Alain Desdoigts, EPEE, Université d'Evry-Val d'Essonne, 4, Boulevard François Mitterrand, 91025 Evry - France. alain.desdoigts@eco.univ-evry.fr.

** Fabien Moizeau, EUREQua, 106-112, Boulevard de 1’Hôpital, 75013 Paris - France. fmoizeau@ univ-paris1.fr.
} 


\section{Introduction}

The pattern of income distribution in a society reflects a history of class bargains and struggles which is specific to each economy. On the other hand, recent years have witnessed that economic clustering and social break-up remain essential features of most economies and that inequality is on average and in most cases harmful for growth. As a consequence, it is often argued that there is a need for state action to arise to limit the degree of group inequality which in turn is expected to enhance growth. In this paper, we shed light on how redistribution or government transfers may (i) modify the community structure of an economy, (ii) generate both intra and intergenerational mobility, (iii) influence accumulation of human capital and the growth process of an economy along its transitional path and the associated steady state. Our framework relies on distributional tensions which act in many subtle ways depending on social affinity and more specifically on the prospect of upward or downward mobility of the different income groups. It provides an explanation as to why government transfers may differ so greatly between countries and displays multiple politico-economic regimes which are supported by new international empirical evidence.

Our modelling is based on two types of arguments which have recently been corroborated with robust empirical evidence. The first argument is the following. In some countries like most European countries, the school-finance system is largely centralized and financed by the government. The aim is to provide the same quality and amount of education to everybody everywhere. In contrast, in others like the United States, education is more privately provided and when it is publicly provided, the level of school funding is mostly determined at the local level. However, in both systems, the parental background and neighborhood attributes remain important factors that determine the individual investment effort into education and its productivity.

Even in Europe, human capital investment in a child still highly depends on the income of his parents. (Evidence from the U.S. is provided in now well-known studies by Solon [39] , and Zimmerman [42] , among others.) In Italy, poor and non-educated families are less likely to invest in the education of their children despite an offer of equal opportunities to attend college and university (see, Checchi et al. [11] ). In France, a manager's child spends on average 7.6 years at university compared to 3.5 for a worker's child (see le Monde, 02/27/2001). More generally, 
the propensity to attend university which is almost free of charge is higher among richer-income individuals and still strongly depends on the family income (see, Galland and Rouault [25] ). Higher-income individuals in Europe therefore benefit in a larger extent of public spending in education than poorer-income individuals do, especially at the highest level of education.

It may be the case even within a centralized and public school-finance system, because poorer-income individuals may not have a good perception of the relationship between income and schooling so that they deliberately choose not to attend college or university (See for instance, Streufert [40] who discusses under which conditions underclass social isolation indeed depresses schooling). It may also be true that the returns to education differ across classes of income so that lower-income individuals hesitate to invest into higher-education (see, Checchi et al., respectively Galland and Rouault, for such an empirical evidence in the Italian, respectively the French case). At the very least, most countries experience the lingering nature of education inequality. We therefore argue in our model and in contrast to Glomm and Ravikumar [27] , and Saint Paul and Verdier [38] , that even in the case of centralized public education, not all individuals obtain the same level of education. Instead, following Fernandez and Rogerson [20], we rather consider educational services as a non rival but partially excludable $\operatorname{good}^{1}$.

Moreover, in all countries, there always exists a set of private and individual alternatives and even in the case of public-provision of education richer individuals may be more willing and able to spend extra effort to ensure that their children are progressing well in school, e.g., they may be more willing to pay high housing prices to benefit from the local externalities (local networks or "social capital") associated with a given neighborhood. In France, $44 \%$ of company heads (30\% of senior managers) children attend private schools. Among all those families whose children attend private schools, $77 \%$ choose this alternative because "the right sort of people go there" (see le Monde, 02/06/2001). These neighborhood effects have been found to play a major role beside parental status in generating positive local feedbacks on wealth accumulation and social mobility (see for instance, Borjas [9], and Cooper et al.

\footnotetext{
1 See also Lloyd-Ellis [29] who emphasizes an empirical finding by Mingat and Tan [30] : in developing countries, an average of 71 percent of the population receives primary schooling, but only receive 22 percent of the resources devoted to education, while the 6 percent of the population who attain higher education receive 39 percent of the resources. On the other hand, Filmer and Pritchett [23] find within a set of 35 countries that educational attainment is strongly positively correlated with the household wealth.
} 
[12] ). As a consequence, the acquisition of human capital can not be considered as a discrete choice where you are able or not to obtain education. In contrast to Perotti [32] and Fernandez \& Rogerson [20] , the models closest to ours, we assume that the costs and benefits of education are endogenous and do not rely on the existence of an exogenous threshold; that is, investment opportunities are divisible. We also assume along the lines emphasized by for instance, Bénabou [5], Durlauf [15] , and Fernandez \& Rogerson [21] that beside parental background, peer-group effects are a key factor which lead the all population of an economy to sort itself into relatively homogeneous communities.

If there are capital market imperfections, it is often argued that a way to fight against underclass social isolation from schooling and at the same time to extend to the largest possible portion of the population the benefits of the local externalities which operate through peer influences, may consist of strengthening the forces that make for greater equality among the initially different socioeconomic classes. There is some scope for raising income of poorer-income individuals through reallocation of existing resources. However, in a democracy, the level of redistribution must be chosen by majority voting, and therefore most countries face the traditional conflicts of economic interest between the different income classes whose degree of altruism both within and across generations is only limited. At first sight, it is very unlikely that the relevant mechanism through which distributional tensions arise only relies, as argued in the standard fiscal policy approach (see for instance, Perotti [32] , Alesina and Rodrik [1] , and Persson and Tabellini [35] ), on the gap between the median and the average incomes. One goal of this paper consists of highlighting different ways through which distributional conflicts among income groups do matter to understand the great dispersion of government transfers across countries.

This leads us to the second argument which partially drives our model and which is based on three robust empirical findings. First, more equal societies have on average higher rates of investment in education which are reflected in higher rates of growth. Second, the effect of transfers on growth across nations has been found to be significantly positive. (See for instance Perotti [33] and [34], Figini [22], and the critical survey by Bénabou [6] .) We therefore require our model to be consistent with this class of models where credit constraints prevent poorer-income individuals to invest into education which in turn is expected to foster economic growth in the presence of diminishing returns. 
(See Glomm and Ravikumar [27], Galor and Zeira [26] , Bénabou [5] , and Durlauf [15] , among many others.)

The third empirical evidence remains more like a puzzle: more unequal countries tend to redistribute less, not more! As Bénabou [7] argues: how is it possible that there is more pressure for redistributive policies and more upward social mobility in Scandinavian countries like for instance Sweden compared to the United States although the former are at the same time more equal? We can also refer to Saint Paul [37] who studies the impact of income inequality on fiscal conservatism. Or, following Bolton and Roland [8], how can we explain that the United Kingdom favors lower taxes and less redistribution, while others like the Netherlands favor higher taxes to protect their welfare state?

In this paper, we first study the redistributive conflicts of interest which may arise in an economy composed of heterogeneous individuals who vote over a level of pure redistribution. But, the balance of power in the political system differs from most previous political economy models where the level of tranfers only depends on the distance between the median voter income and the average income in the economy. Our model also reflects a world in which neighborhood attributes and family backgrounds are important in determining the individual investment effort and the returns on education. This allows us to provide an explanation to the above puzzle; that is, why inequality can be associated with less, rather than more, redistributive taxation within some range of inequality? Second, we characterize both the growth process and the income distribution dynamics along the transitional path to the steady state for any given initial pattern of income distribution.

More specifically, we focus on an economy initially composed of three different income classes. Investment in education is not modelled as an exogenous discrete choice. Instead, the investment effort into education of an individual depends on her posttax income. A key feature of our model is that once a lower-income class reaches a threshold level of development such that it becomes homogeneous enough to the next higher-income class, they can form a community in which the lower type experiences a peer-group effect. This threshold level depends on the characteristics of all the members of the community.

The local connection which causes the formation of relatively homogeneous communities has been analyzed in now seminal papers by Bénabou [4] , and Fernandez and Rogerson [21], among others. In both these studies, authors 
focus on games where agents first choose the community in which they will live and then decide on how much to spend on education which is publicly provided at the community level through local taxation. Our model combines both the endogenous fiscal policy and the local connection approaches. It also consists of a two-stage game but where agents first vote over a level of pure redistribution which modifies the income distribution and more specifically, which affects how the entire economy breaks up or stratifies into communities. In the second stage, the level of educational services available to each agent is determined where all agents of a same community benefit from a similar level of quasi-public resources and where the richer the community is on average the higher is this level. Moreover, there is an explicit local trickle down mechanism which reflects for instance the social networks built by higher-income individuals and which become available to those poorer-income agents who eventually live in the same community.

The possibility either to form clubs or to block them within a pure redistribution framework, and endogenizing the opportunity cost of education yield conclusions which are more intricate compared to the existing and associated literature. Suppose that the middle-income class is well-aware that redistribution would allow them to benefit from higher local externalities like social networks as they might be available to the high-income class or to be more able and willing to attend college or university whatever the latter is privately or publicly provided. Then, what matters for the former is its prospect of upward social mobility; that is, to eventually be able to be affiliated to the community of the higher-income individuals, and not only whether it is richer or poorer relative to the mean as argued in standard political economy models. Even though the low-income class may be excluded from this new community, as long as it is better off with that level of redistribution compared to any lower tax rate, the political outcome which prevails is the one most preferred by the middle-income class. However, there also might exist ex ante levels of inequality such that the low-income class opposes the high levels of redistribution preferred by the middle-income class. This may occur in some range of inequality where the pressure for redistribution most preferred by the middle-income class leads the low-income class to lag further behind the two other income classes while they could catch up with a lower level of redistribution. Notice that within our majority voting framework, these conflicts of interest can also give rise to Condorcet cycles. 
The analysis of the conflicts of interest across our three initially differently endowed income classes allows us to infer that: (i) the pressure for redistributive policies is not necessarily smaller in a rich economy where the income of the middle class is high relative to the average compared to the pressure that prevails in a poorer economy where the income of the middle class may be smaller relative to the average, (ii) there exist in some range of inequality, political equilibria where both the low- and the high-income classes agree on a similar level of redistribution which is smaller than the one most preferred by the middle-income class, (iii) in a completely segregated economy, a rise in the wealth bias against the poor or an increase in the Gini coefficient may lead to lower the equilibrium level of redistribution, (iv) finally and more generally, more unequal and segmented economies may redistribute less, not more. Although it is certainly not appropriate to draw definitive conclusions because of contemporary data and sample size problems, we also provide international empirical evidence that tends to support these results and more particularly the nature of the redistributional tensions and the existence of multiple politico-economic regimes as identified and discussed below in the text.

The analysis would not be complete without considering the dynamics of inequality and the growth process underlying our modelling. Our model displays multiple history-dependent steady states, describing either a situation of equality and integration or segregation with persistent inequality. Along the transitional path, a large range of initial conditions may lead economies to cross Condorcet cycles regions. Finally, introducing redistribution increases the number of candidates for the integrated equilibrium and fosters growth.

The paper is organized as follows. Section 2 presents the structure of the model. In Section 3, we define what is an equilibrium partition and provide information about the key conflicts of interest which may occur depending on the initial pattern of income distribution. We also examine under which conditions multiple politico-economic regimes may occur and provide international empirical evidence. Section 4 discusses the dynamic properties of the model. Finally, Section 5 concludes and most of the technical apparatus is left to the appendix. 


\section{The Model}

Our objectives are similar to those of Saint Paul [37] and Bénabou [7] although our analytical approach differs greatly. It proposes an alternative explanation as to why the pattern of income distribution across nations can be associated with multiple politico-economic regimes.

Our modelling strategy consists in a two-stage game which is repeated every period and the ingredients of the model which allow us to explore this puzzling feature across nations are: (i) a level of pure redistribution determined in a first stage by majority vote and which involves deadweight losses as for instance in Perotti [32] , and Bolton and Roland [8] (ii) which modifies the community structure of the whole economy and gives rise to a decentralized formation of clubs of agents who differ only with respect to their posttax income, (iii) and who decide in the second stage of the game on how much to invest into skill-acquisition, (iv) a level of educational services that depend on the characteristics of the community's members and which are distributed uniformly to people in the affiliational club. Non members are outliers and may partially be excluded from its benefits. (v) Finally, both the parental background and those quasi-public resources drive accumulation of human capital (see Bénabou [4] and [5] ). Note that for a given level of redistribution, the equilibrium partition which emerges in the absence of Condorcet cycles belongs to the core; that is, there is no other partition of the economy which is Pareto-improving.

\subsection{Preferences, Technologies, and the Tax System}

We consider an infinity of non-overlapping generations, each living one period. Let there be a large number of individuals $i \in \Omega$ within each generation, and total population $\Omega$ lives in a world composed of three groups of agents of equal $\operatorname{size}^{2} n$, and characterized by different pre-tax human capital endowment, $h_{t}^{i}$, with $i=h, m$, or $l$, which is the only source of heterogeneity. At $t=0$, pre-tax human capital endowments are characterized by the following inequalities: $0<h_{0}^{l}<h_{0}^{m}<h_{0}^{h}$.

Agents may group themselves into clubs that are defined as follows: 2 In fact, all what we require to solve the model is that no single class is able to impose its most preferred outcome on the others and that the size
of two income classes amounts at least to fifty percent of the entire population. 
Definition 1: A nonempty subset $S_{j t}$ of $\Omega$ is called a club or a community and $P_{t}=\left(S_{1 t} ; \ldots ; S_{J t}\right)$ for $j=1, \ldots ., J$, is called a partition of $\Omega$ if:

(1) $\bigcup_{j=1}^{J} S_{j t}=\Omega$;

(2) $S_{j t} \bigcap S_{j^{\prime} t}=\emptyset$ for $j \neq j^{\prime}$.

Each agent is affiliated to one and only one community.

Following Saint Paul and Verdier [38] , each individual maximizes a strictly concave utility function of the form

$$
U\left(c_{j, t}^{i}, h_{j, t+1}^{i}\right)=\ln c_{j, t}^{i}+\ln h_{j, t+1}^{i}
$$

where $c_{j, t}^{i}$ is the consumption level of individual $i$ at time $t$ and $h_{j, t+1}^{i}$ is the human capital stock left to her offspring when she belongs to the community $S_{j t}$.

Individuals have no access to credit. They accumulate human capital as follows:

$$
h_{j, t+1}^{i}=\kappa \cdot G_{j t}^{\beta} \cdot \bar{h}_{t}^{\alpha} \cdot\left(h_{t}^{i}\right)^{1-\alpha-\beta}
$$

Following Bénabou [5], the accumulation of human capital reflects the influence of family through the pre-tax human capital inherited from parents $\left(h_{t}^{i}\right)$, the influence of economy-wide knowledge spillovers through $\bar{h}_{t}$ which is the average stock of human capital in the entire society at time $t$. Finally, agents are also concerned about the level of quasi-public resources supplied in the club $\left(G_{j t}\right)$ and which are provided uniformly to people of the same community. We assume that $\alpha, \beta$, and $1-\alpha-\beta \in(0,1)$ so that all factors exhibit diminishing returns.

Let $G_{j t}$ be the quantity of public good available in the club $S_{j t}$. Following Bahram et al. [2], we assume that $G_{j t}$ is the sum of the individual investment efforts in education of the members of the community, denoted by $g_{j t}^{i}$, given some linear congestion costs denoted by $a$.

$$
G_{j t}=\sum_{i \in S_{j t}} g_{j t}^{i}-n_{j t} a
$$

where $n_{j t}$ is the number of agents in the community $S_{j t}$.

Notice that although $G_{j t}$ will be determined within a non cooperative game, this simple formulation allows us to 
shed light on how the community structure which emerges from the first stage of the game interacts with the quality of education across communities and the individual investment effort into education as well as to incorporate all the above discussed ingredients in an easily tractable fashion.

At the beginning of the period agents vote over the level of income taxes that are proportional to pre-tax income. Total tax revenues are redistributed as a lump sum transfer that is constant across individuals. The task of redistribution is therefore to modify economic disparities across heterogeneous groups of persons. The government budget is always balanced. We also assume that taxation involves deadweight losses. More specifically, there are convex costs in collecting taxes: if $\tau$ is the tax rate, $\tau \bar{h}_{t}$ is collected but only $\left(\tau-\tau^{2}\right) \bar{h}_{t}$ can be redistributed to each individual.

\subsection{Optimal Individual Investment Effort into Education}

The model is solved by working backwards from the second stage of the game. We first determine the optimal individual investment effort into education given her posttax income and the equilibrium community structure of the economy which emerges from the first stage of the game. The political equilibrium and the process of club formation is discussed in the next section.

The choice of individual $i$ 's effort of investment into education $g_{j t}^{i}$ when she belongs to a community $S_{j t}$ is found by maximizing

$$
\begin{aligned}
& U\left(c_{j, t}^{i}, h_{j, t+1}^{i}\right)=\ln c_{j, t}^{i}+\ln h_{j, t+1}^{i} \\
& \text { subject to : } \\
& c_{j, t}^{i}+g_{j t}^{i} \leq(1-\tau) h_{t}^{i}+\left(\tau-\tau^{2}\right) \bar{h}_{t} \\
& h_{j, t+1}^{i}=\kappa \cdot G_{j t}^{\beta} \cdot \bar{h}_{t}^{\alpha} \cdot\left(h_{t}^{i}\right)^{1-\alpha-\beta} \\
& G_{j t}=\sum_{i \in S_{j t}} g_{j t}^{i}-n_{j t} a \\
& g_{j t}^{i} \geq 0 \text {, given } h_{t}^{i}, \bar{h}_{t} \text {, and } \tau \text {. }
\end{aligned}
$$

Each agent faces the same trade off. Her posttax income is devoted either to private consumption or to investment into education. Further, the chosen optimal individual investment effort is such that a threshold level of after-tax income 
is necessary for an agent to be able to benefit from the level of public good provided in the community $S_{j t}$. It is such that

$$
g_{j t}^{i}=\max \left\{0,(1-\tau) h_{t}^{i}+\left(\tau-\tau^{2}\right) \bar{h}_{t}-\frac{1}{\beta} G_{j t}\right\}
$$

Literally, the higher the posttax income of an agent in a given community, the higher the level of her investment into education in that community. The posttax income does not determine whether an individual does acquire education or not, it rather determines how much education she will receive which is given by

$$
G_{j t}=\frac{\beta}{\beta+n_{j t}} \sum_{i \in S_{j t}}\left((1-\tau) h_{t}^{i}+\left(\tau-\tau^{2}\right) \bar{h}_{t}-a\right)
$$

Note that not all agents of a same club need to contribute to the same extent to the provision of the public good available in that club. Instead, two types of agents may belong to the same community where both types experience a peer group effect. On the one hand, the equilibrium level of $G_{j t}$ reflects the traditional effect where a low-income class (or the weak students) may derive more benefit from educational spending when higher-income individuals (or strong students) are present in the community (classroom). On the other hand, richer communities benefit from higher levels of educational spending.

Replacing the expression of $G_{j t}$ into the above individual $i$ 's utility function, we get the following indirect utility function

$$
V^{i}\left(S_{j t} ; \tau\right)=(1+\beta) \ln G_{j t}+\ln \left(\beta^{-1} \cdot \kappa \cdot \bar{h}_{t}^{\alpha} \cdot\left(h_{t}^{i}\right)^{1-\alpha-\beta}\right)
$$

At this stage, it is worth noticing that the second term in the sum is determined at the beginning of the period and therefore at the beginning of the game for each individual. Hence, all that matters to compute the utility gain or loss of the different agents after redistribution is the level of public good available in the club to which they belong; that is, $\ln G_{j t}$ which reflects the capacity or ability of the individual to afford the costs involved in education through her posttax income but also the incentive to do so through her affiliational to a community.

\section{Equilibrium Partition, Preferred Tax Rate, and Political Outcome}

We now move back up the game tree. First, we define what is an equilibrium partition. Second, we provide information 
about the trade-off faced by the different income classes and the conflicts of interest which may arise for any given ex ante pattern of income distribution. Third, we solve the first stage of the game and determine both the political equilibrium if it exists ${ }^{3}$, and the associated equilibrium partition.

\subsection{Equilibrium Partition}

The first stage of the game consists of determining the community structure which emerges from the political equilibrium given the after-tax income distribution. We require that any equilibrium partition, denoted $P_{t}^{E}$, of agents into clubs be in the core. That is, there exists no other group that could block $P_{t}^{E}$ (see for instance, Farrell and Scotchmer [19] ).

It then follows that because of the existence of crowding costs linked to the size of a club, an agent of type $i$ belongs to a community $S_{j t}^{E}$ of the equilibrium partition if her posttax income has reached a threshold level such that she is able to invest into education a strictly positive amount of her posttax income in that community. From equations (4) and (5), for an agent of type $i$ in $S_{j t}^{E}$, this condition yields the following inequality:

$$
(1-\tau) h_{t}^{i}+\left(\tau-\tau^{2}\right) \bar{h}_{t}>\frac{1}{\beta+n_{j t}} \sum_{i \in S_{j t}^{E}}\left((1-\tau) h_{t}^{i}+\left(\tau-\tau^{2}\right) \bar{h}_{t}-a\right)
$$

In other words, no non contributing individual can be accepted in a community $S_{j t}^{E}$ of the equilibrium partition. Consider a club in a partition in which at least one of its members does not contribute. This agent increases the congestion cost. Obviously, all the contributors of this club are better off by excluding this agent. Hence, this partition is blocked by the set of contributors and is not an equilibrium partition. Notice that preferences across agents are homogeneous and that the marginal congestion cost is constant. When an agent of a given type is able to positively contribute to the provision of the public good in a given community all members in that community are better off when all agents of the same type join that club. An equilibrium partition is such that all agents of a same type belong to the same club. Hence, we focus on the heterogeneity within communities rather than on their sizes. Finally, the clubs in the equilibrium partition are consecutive. Suppose they are not and let us consider the partition $P_{t}=(\{h, l\} ;\{m\})$,

\footnotetext{
3 The final decision about the tax rate is traditionally taken by majority rule; that is, the only admissible outcomes are those which defeat every other in a binary majority contest (see for instance, Moulin [31]).
} 
$P_{t}$ is blocked by $\{h, m\}$ because both the high and the middle-income classes are strictly better off in this new club.

Therefore, we define the partitions which are candidates for the core of the economy as,

Definition 2: At each date, if it exists, the equilibrium partition is one of these four cases:
(i) $P_{t}^{I}=(\{h, m, l\})$,
(ii) $P_{t}^{I I}=(\{h, m\} ;\{l\})$,
(iii) $P_{t}^{I I I}=(\{h\} ;\{m\} ;\{l\})$,
(iv) $P_{t}^{I V}=(\{h\} ;\{m, l\})$

\subsection{Conflicts of Interest over Tax Rates}

A first set of relevant tax rates which must be considered to determine the political equilibrium at each time $t$, is the set of tax rates that maximize an agent $i$ 's indirect utility function or equivalently the level of quasi-public resources provided in the community she may have access to after redistribution. It is defined as ${ }^{4}$

$$
\lim _{a \rightarrow 0} \tau_{S_{j t}}^{*}=\max \left\{0, \arg \max \left(\frac{\beta}{n_{j t}+\beta} \sum_{i \in S_{j t}}(1-\tau) h_{t}^{i}+\left(\tau-\tau^{2}\right) \bar{h}_{t}\right)\right\}
$$

and each corresponding community's optimal tax rate is

$$
\lim _{a \rightarrow 0} \tau_{S_{j t}}^{*}=\max \left\{0, \frac{1}{2}\left(1-\frac{\sum_{i \in S_{j t}} h_{t}^{i}}{n_{j t} \bar{h}_{t}}\right)\right\}
$$

Notice that the definition of $\tau_{S_{j t}}^{*}$ is such that whatever the ex ante income distribution is, we always have the following ranking of tax rates:

$$
0=\tau_{\{h\}}^{*}=\tau_{\{h, m\}}^{*}=\tau_{\{h, m, l\}}^{*} \leq \tau_{\{m\}}^{*}<\tau_{\{m, l\}}^{*}<\tau_{\{l\}}^{*}<\frac{1}{2}
$$

Agents in poorer communities tend to favor higher tax rates, while the members affiliated to richer neighborhoods have their well-being maximized at lower tax rates. This reflects the traditional conflict of interest between rich and poor.

Second, as in Perotti [32] and Fernandez and Rogerson [20] , various discontinuities arise in the indirect utility functions of both the middle and the low-income groups which lead their preferences in some range of inequality not to be single-peaked. Indeed, for a given pattern of ex ante income distribution, there might exist a redistributive policy which modifies the community structure of the economy and leads a lower-income class to join a higher initially endowed income group. In that case and in the absence of serious distortions, the benefits for the former are straight-

\footnotetext{
4 From now on, we assume without loss of generality that congestion costs are infinitesimal.
} 
forward as the level of quasi-public resources or the social capital available in this new community is enhanced by the presence of the other group.

For instance, the level of redistribution required for the low-income group to join the middle-income class so that they form a Pareto-improving community $S_{j t}=\{m, l\}$ compared to the situation where they both remain on their own for the same level of redistribution, is defined as follows:

$$
V^{m}\left(\{m, l\} ; \tilde{\tau}_{\{m, l\}}\right)=V^{m}\left(\{m\} ; \tilde{\tau}_{\{m, l\}}\right)>V^{l}\left(\{l\} ; \tilde{\tau}_{\{m, l\}}\right)
$$

Using equation (7), $\tilde{\tau}_{\{m, l\}}$ tends to

$$
\lim _{a \rightarrow 0} \widetilde{\tau}_{\{m, l\}}=\max \left\{0, \frac{n h_{t}^{m}-(n+\beta) h_{t}^{l}}{\beta \bar{h}_{t}}\right\}
$$

$\tilde{\tau}_{\{m, l\}}$ is therefore defined as the marginal tax rate such that the middle-income class is indifferent between the two clubs $\{m\}$ and $\{m, l\}$.

Similarly, we must also consider the following relevant candidates:

$$
\begin{gathered}
\lim _{a \rightarrow 0} \widetilde{\tau}_{\{h, m\}}=\max \left\{0, \frac{n h_{t}^{h}-(n+\beta) h_{t}^{m}}{\beta \bar{h}_{t}}\right\} \\
\lim _{a \rightarrow 0} \widetilde{\tau}_{\{h, m, l\}}=\max \left\{0, \frac{n\left(h_{t}^{h}+h_{t}^{m}\right)-(2 n+\beta) h_{t}^{l}}{\beta \bar{h}_{t}}\right\}
\end{gathered}
$$

where $\tilde{\tau}_{\{h, m\}}$, respectively $\tilde{\tau}_{\{h, m, l\}}$, are the tax rates such that $V^{h}\left(\{h, m\} ; \tilde{\tau}_{\{h, m\}}\right)=V^{h}\left(\{h\} ; \tilde{\tau}_{\{h, m\}}\right)$, respectively $V^{i}\left(\{h, m, l\} ; \widetilde{\tau}_{\{h, m, l\}}\right)=V^{i}\left(\{h, m\} ; \widetilde{\tau}_{\{h, m, l\}}\right)$, for $i=h, m^{5}$.

Literally, from equation (11), when the ex ante pattern of income distribution is such that $n\left(h_{t}^{h}+h_{t}^{m}\right)-(2 n+\beta) h_{t}^{l}<$ 0 , no redistribution is needed for the partition $P_{t}^{I}=(\{h, m, l\})$ to emerge. The whole economy is homogeneous enough so that the three income classes are able to attain equal educational opportunities. The same result applies for $P_{t}^{I I}=(\{h, m\},\{l\})\left(P_{t}^{I V}=(\{h\} ;\{m, l\})\right)$ when the initial income distribution is such that $n h_{t}^{h}-(n+\beta) h_{t}^{m}<0$ $\left(n h_{t}^{m}-(n+\beta) h_{t}^{l}<0\right)$. In that case, no redistribution is needed for the middle- (low-) income group to join the high(middle-) income group. Notice that as long as the ex ante distribution is such that $\widetilde{\tau}_{\{h, m\}} \leq \widetilde{\tau}_{\{m, l\}}$, then $\tilde{\tau}_{\{h, m\}}$ is

\footnotetext{
5 Notice that the tax rate $\tau$ such that $V^{h}(\{h\} ; \tau)=V^{h}(\{h, m, l\} ; \tau)$ is an irrelevant candidate. Indeed, whatever the level of inequality, we have $\tilde{\tau}_{\{h, m\}}<\tau$. Hence, the community $\{h, m\}$ already exists with levels of redistribution smaller than $\tau$ and the above trade off is therefore irrelevant.
} 
preferred by a majority in pairwise comparison to $\tilde{\tau}_{\{m, l\}}$. This reflects our assumption that a positive intra community externality is exerted by the relatively higher-income individuals on the poorer-income ones.

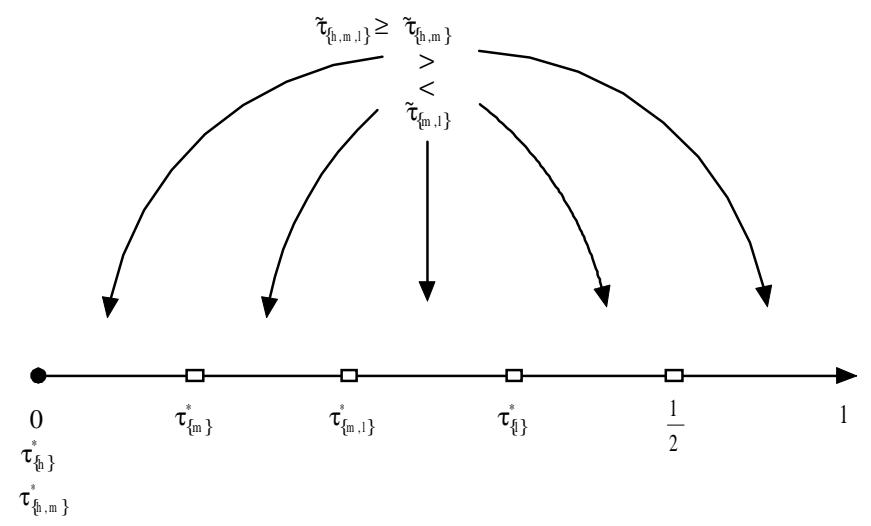

Figure 1: The relative position of the different candidates provide information about the initial pattern of inequality.

In our discussion, $\tau_{S_{j t}}^{*}$ corresponds to the tax rate which maximizes the level of public good in the club $S_{j t}$. However, for a given initial distribution, this tax rate may not be large enough for the corresponding community to endogenously form. As a consequence, the political outcome depends on the relative levels of all the tax rates which are candidates for the political equilibrium; that is, $\widetilde{\tau}_{S_{j t}}$ and $\tau_{S_{j t}}^{*}$ (see Figure 1).

Consider the trade off for an agent who initially belongs to the middle-income group such as displayed in Figure 2; the tax rate that maximizes her utility function if she forms a club only with agents of the same kind is $\tau_{\{m\}}^{*}$. Note that a small increase in the tax rate causes a reduction in her utility. However, further increase in the tax rate until $\widetilde{\tau}_{\{h, m\}}$ allows her to have access to a richer community. As shown in Figure 2, the middle-income class is now better off by forming a club with agents of type $h$. It most prefers $\widetilde{\tau}_{\{h, m\}}$ to $\tau_{\{m\}}^{*}$ and the former is permissible as long as the level of distortions associated with this fiscal policy remains reasonable; that is, $\widetilde{\tau}_{\{h, m\}} \leq 1 / 2$. More generally, a jump in the indirect utility function of both the middle- and the low-income groups arises whenever the tax rate reaches a level such that one of these groups' posttax income becomes large enough to form a relatively homogeneous community with the next higher-income class. This leads their preferences not to be single-peaked in some range of inequality. 


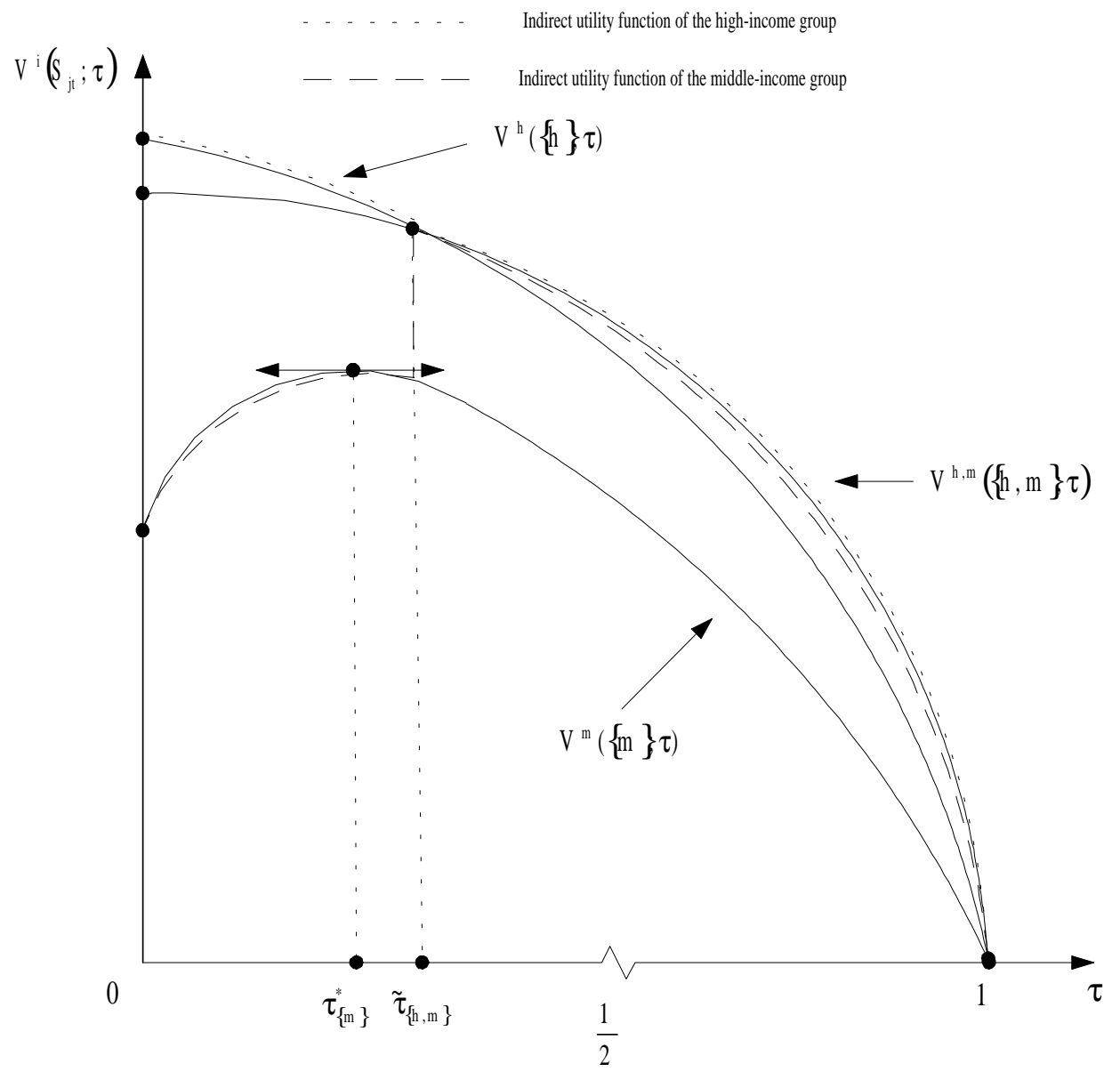

Figure 2: Indirect utility functions for the $\{h, m\}$, and $\{h\}$, and $\{m\}$ communities. A discontinuity arises in the utility of an agent $m$ when the tax rate reaches the marginal level $\tilde{\tau}_{\{h, m\}}$. The display of the indirect utility function of the different income groups only considers that part of $V^{i}\left(S_{j t} ; \tau\right)$ which depends on $G_{j t}$. 


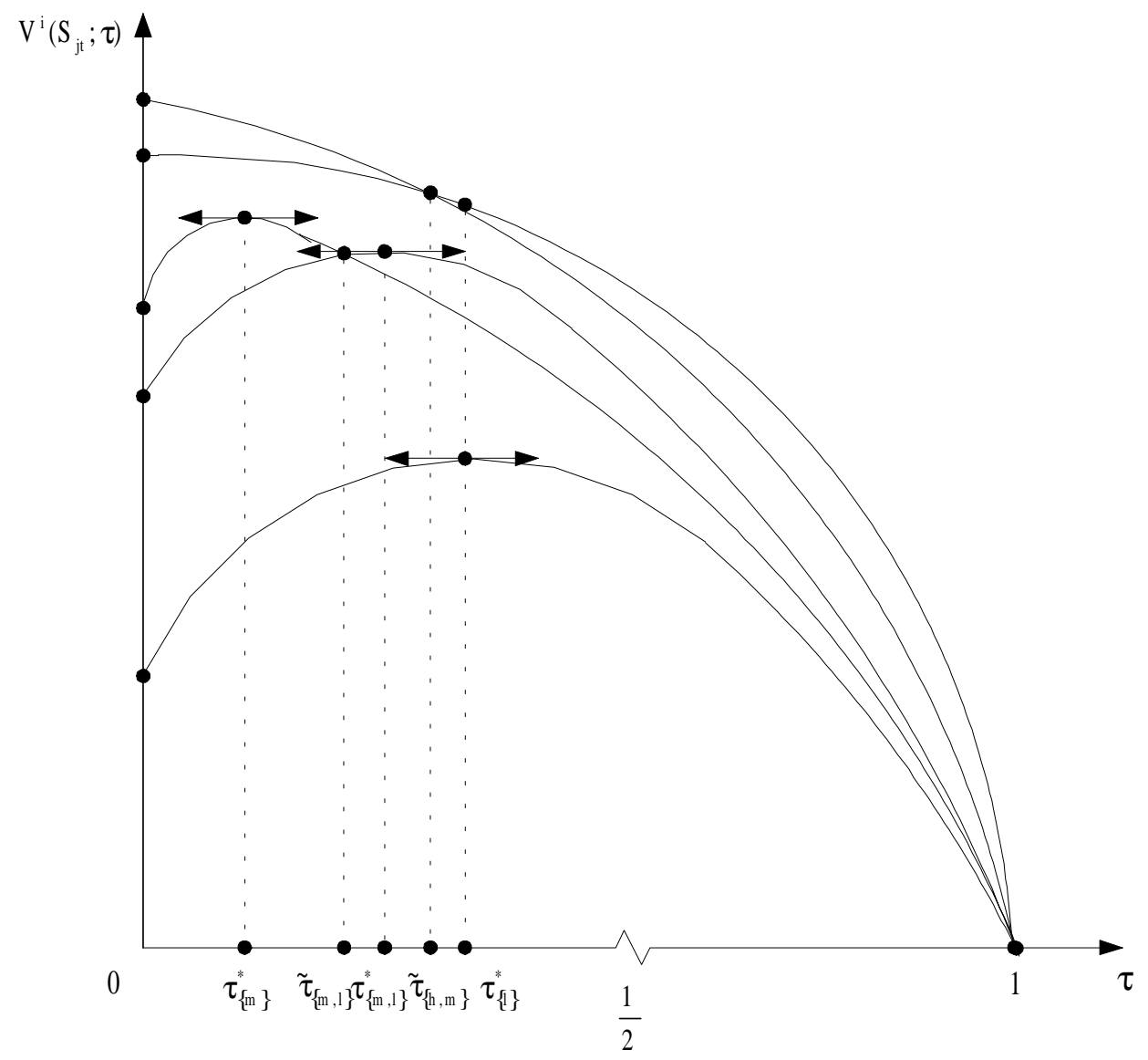

Figure 3: Indirect utility functions for the $\{h, m\},\{m, l\},\{h\},\{m\}$, and $\{l\}$ communities. An initial pattern of income distribution that yields a Condorcet paradox. The display of the indirect utility function of the different income groups only considers that part of $V^{i}\left(S_{j t} ; \tau\right)$ which depends on $G_{j t}$. 
Because of non single peakedness, the usual conditions for the existence of a stable majority cannot be applied directly and the most preferred tax rate in the economy is not always associated with the agent of type $m$ 's most preferred tax rate. The low-income class may in some cases, depending on the initial pattern of income distribution, prefer a lower tax rate compared to the preferred tax rate by the middle-income group. An example as displayed in Figure 3 where we consider the indirect utility functions of agents $h, m$, and $l$, illustrates the possibility for Condorcet paradoxes to arise given a particular initial pattern of income distribution. Consider the following profile of relevant preferences for the three income classes associated with the income distribution as depicted in Figure 3:

$$
\begin{gathered}
V^{h}\left(\{h\} ; \tau_{\{m\}}^{*}\right)>V^{h}\left(\{h\} ; \tau_{\{m, l\}}^{*}\right)>V^{h}\left(\{h, m\} ; \tilde{\tau}_{\{h, m\}}\right)>V^{h}\left(\{h, m\} ; \tau_{\{l\}}^{*}\right) \\
V^{m}\left(\{h, m\} ; \widetilde{\tau}_{\{h, m\}}\right)>V^{m}\left(\{h, m\} ; \tau_{\{l\}}^{*}\right)>V^{m}\left(\{m\} ; \tau_{\{m\}}^{*}\right)>V^{m}\left(\{m, l\} ; \tau_{\{m, l\}}^{*}\right) \\
V^{l}\left(\{m, l\} ; \tau_{\{m, l\}}^{*}\right)>V^{l}\left(\{l\} ; \tau_{\{l\}}^{*}\right)>V^{l}\left(\{l\} ; \tilde{\tau}_{\{h, m\}}\right)>V^{l}\left(\{l\} ; \tau_{\{m\}}^{*}\right)
\end{gathered}
$$

It is easily checked that there is no Condorcet winner. Therefore, in contrast, for instance, to Perotti [32] , initial patterns of income distribution may occur so that there is no value of $\tau$ which is stable against the rule of majority.

\subsection{Political Equilibrium}

Let us start the discussion by recalling that levels of inequality where $\frac{h_{t}^{h}}{h_{t}^{m}} \leq 1+\left(\frac{n}{\beta}\right)^{-1}$ or $\frac{h_{t}^{m}}{h_{t}^{l}} \leq 1+\left(\frac{n}{\beta}\right)^{-1}$; that is $\tilde{\tau}_{\{h, m\}}=0$, or $\widetilde{\tau}_{\{m, l\}}=0$, are such that no redistribution is needed for either the middle- or the low-income class to form a club with respectively, either the high- or the middle-income group. Then, we can easily conclude that without redistributive policies, as soon as $\frac{h_{t}^{h}}{h_{t}^{m}}>1+\left(\frac{n}{\beta}\right)^{-1}$, and $\frac{h_{t}^{m}}{h_{t}^{l}}>1+\left(\frac{n}{\beta}\right)^{-1}$ the equilibrium partition is $P_{t}^{E}=(\{h\} ;\{m\} ;\{l\})$. Note also that without redistribution, the conditions on the pattern of relative inequality between the three income groups required for the all economy to benefit from the same level of public good is even more restrictive. Indeed, it is such that $\frac{h_{t}^{h}}{h_{t}^{m}} \leq\left(2+\left(\frac{n}{\beta}\right)^{-1}\right)\left(\frac{h_{t}^{m}}{h_{t}^{2}}\right)^{-1}-1$.

Therefore, two questions arise: what are the consequences of introducing a pure redistributive scheme on the formation of economic communities; that is, on the stratification of the income distribution? And, what is the impact of inequality on redistribution both within and across the different equilibrium partitions? In a world where an individual's 
capacity to invest in education and to benefit from high local spillovers or from higher educational services depends on her posttax income, it turns out that the link between inequality and redistribution is more complex than advocated in conventional models of political economy. We now highlight the role of income distribution in setting the uneven levels of redistribution across nations.

Propositions 1 to 5 characterize for any ex ante pattern of income distribution the tax rate which is chosen by a majority and the equilibrium economic partition which emerges from the elected redistributive policy as well as the range of inequality where Condorcet cycles are likely to emerge. The following propositions are better illustrated in Figure 4 which displays the ranges of ex ante inequality where the different equilibrium partitions emerge (some information about the construction of this figure is provided in Appendix $\mathrm{B}^{6}$ ). Whenever there is a Condorcet winner, the associated equilibrium partition is unique and it belongs to the core.

The following proposition describes Region $I$ displayed in Figure 4.

Proposition 1. First, the equilibrium partition $P_{t}^{E}=(\{h, m, l\})$ occurs when the posttax income inequality between $h_{t}^{h}, h_{t}^{m}$, $h_{t}^{l}$ is such that $n\left(h_{t}^{h}+h_{t}^{m}\right)-(2 n+\beta) h_{t}^{l}<0$. In that case, the whole population agrees to vote in favor of no redistribution. Second, $P_{t}^{E}=(\{h, m, l\})$ is also the equilibrium partition when both the low- and the middle-income classes are similarly endowed $\left(h_{t}^{m}=h_{t}^{l}\right)$ and as long as they are both better off in a community $\{h, m, l\}$ rather than a community $\{m, l\}$. In that case, the most socially preferred tax rate is $\tilde{\tau}_{\{h, m, l\}} \geq 0$.

\section{Proof. See Appendix A}

Rather naturally, redistribution increases the range of ex ante relative inequality where the grand coalition belongs to the core. The three other possible equilibrium partitions at least require that $n\left(h_{t}^{h}+h_{t}^{m}\right)-(2 n+\beta) h_{t}^{l} \geq 0$. The first one, depicted in Region $I I$ of Figure 4, is described in the following proposition,

\footnotetext{
${ }_{6} A A \equiv V_{t}^{m}\left(\{m\} ; \tau_{\{m\}}^{*}\right)=V_{t}^{m}\left(\{h, m\} ; \tilde{\tau}_{\{h, m\}}\right) \cdot B B \equiv V_{t}^{l}\left(\{l\} ; \tau_{\{m\}}^{*}\right)=V_{t}^{l}\left(\{l\} ; \tilde{\tau}_{\{h, m\}}\right) \cdot C C \equiv V_{t}^{m}\left(\{m\} ; \tau_{\{m\}}^{*}\right)=$ $V_{t}^{m}\left(\{m, l\} ; \tau_{\{m, l\}}^{*}\right) \cdot D D \equiv V_{t}^{m}\left(\{m, l\}, \tilde{\tau}_{\{h, m\}}\right)=V_{t}^{m}\left(\{m\}, \tau_{\{m\}}^{*}\right) . E E \equiv V_{t}^{i}\left(\{m, l\} ; \tilde{\tau}_{\{h, m\}}\right)=V_{t}^{i}\left(\{h, m, l\} ; \tilde{\tau}_{\{h, m, l\}}\right)$ for $i=m, l . F F \equiv V_{t}^{i}\left(\{m, l\} ; \tau_{\{m, l\}}^{*}\right)=V_{t}^{i}\left(\{h, m, l\} ; \tilde{\tau}_{\{h, m, l\}}\right)$ for $i=m, l$.
} 


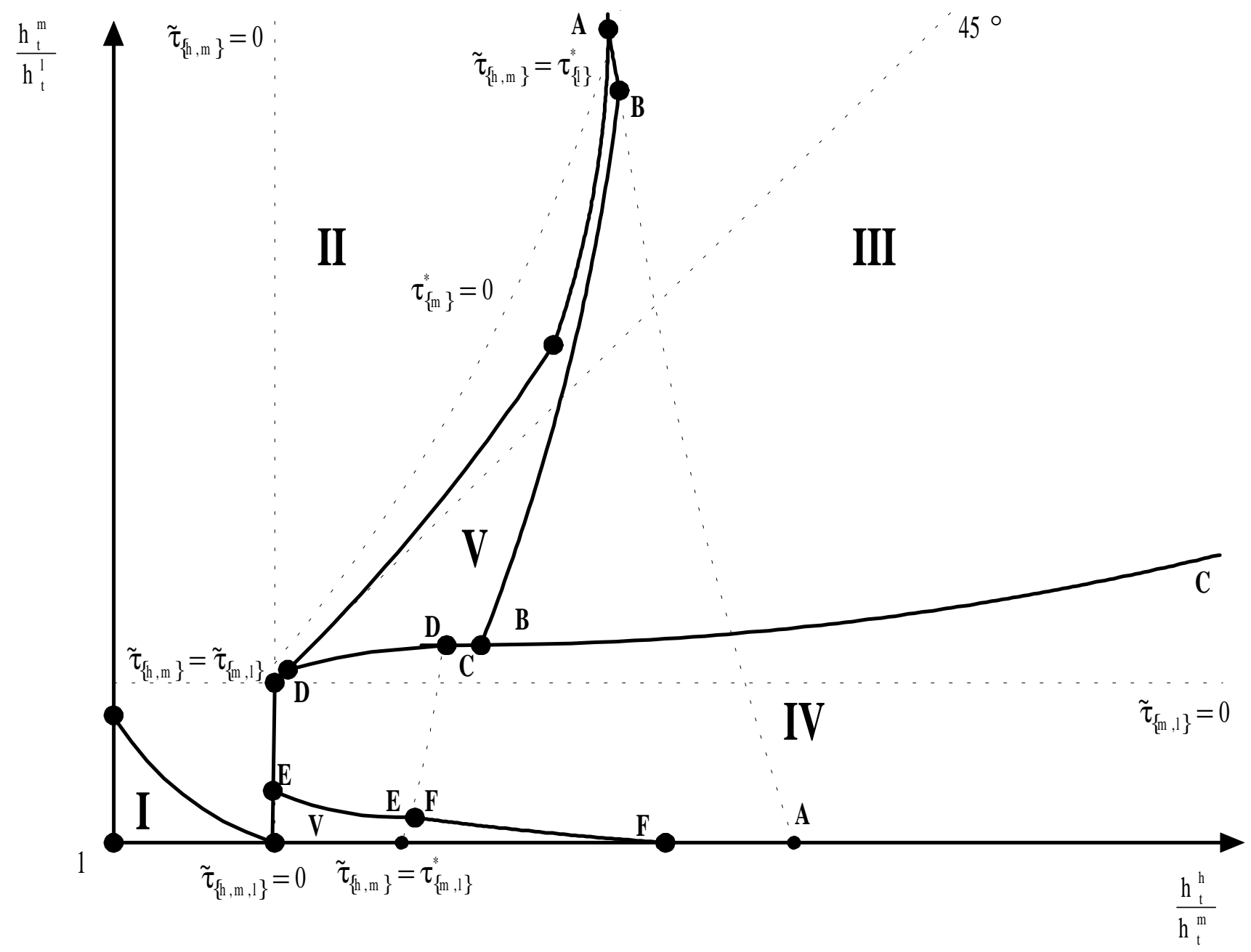

Figure 4: Equilibrium partition regions in the plane $\left(\frac{h_{t}^{h}}{h_{t}^{m}}, \frac{h_{t}^{m}}{h_{t}^{L}}\right)$. Region $I$ corresponds to the equilibrium par-

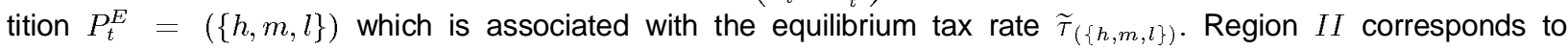
$P_{t}^{E}=(\{h, m\} ;\{l\})$ and either $\tau_{\{h, m\}}^{*}$ or $\tilde{\tau}_{\{h, m\}}$. Region $I I I$ corresponds to $P_{t}^{E}=(\{h\} ;\{m\} ;\{l\})$ and $\tau_{\{m\}}^{*} \geq 0$. Region $I V$ corresponds to $P_{t}^{E}=(\{h\} ;\{m, l\})$ and either $\tau=\left(n h^{h}-(n+\beta) h^{m}\right) / \beta \bar{h}$ or $\tau_{\{m, l\}}^{*}$. Finally, Region $V$ depicts ranges of inequality where there are Condorcet cycles. Further information about the construction of this figure is available in Appendix B. 
Proposition 2. When the ex ante pattern of relative income inequality between $h_{t}^{h}, h_{t}^{m}, h_{t}^{l}$ is such that the three following conditions are satisfied simultaneously: (i) the marginal tax rate required for the middle-income class to form a club with the high-income class is smaller or equal to the tax rate required for the low-income group to join the middle-income one $\left(0 \leq \widetilde{\tau}_{\{h, m\}} \leq \widetilde{\tau}_{\{m, l\}}\right)$, (ii) $\widetilde{\tau}_{\{h, m\}}$ is smaller or equal to the most preferred tax rate by the low-income class when it remains on its own $\left(\tau_{\{l\}}^{*}\right)$, (iii) the middle-income class is better off by forming a club with the high-income class rather than remaining on its own; that is, $V_{t}^{m}\left(\{h, m\} ; \tilde{\tau}_{\{h, m\}}\right) \geq$ $V_{t}^{m}\left(\{m\} ; \tau_{\{m\}}^{*}\right)$, the equilibrium partition is $P_{t}^{E}=(\{h, m\} ;\{l\})$ and a majority emerges in favor of $\tilde{\tau}_{\{h, m\}} \geq 0$, whatever $h_{t}^{m} \gtreqless \bar{h}_{t}$.

Proof. See Appendix A

Region $I I$ in the plane $\left(\frac{h_{t}^{h}}{h_{t}^{m}}, \frac{h_{t}^{m}}{h_{t}^{l}}\right)$ is always located above the $45^{\circ}$-line when $\widetilde{\tau}_{\{h, m\}}>0$; that is, for the equilibrium partition $(\{h, m\} ;\{l\})$ to emerge, the economy must initially exhibit a clear wealth bias against the poor. Notice that by definition, when $\widetilde{\tau}_{\{h, m\}}>0$, it increases with both $\frac{h_{t}^{h}}{h_{t}^{m}}$ and $\frac{h_{t}^{m}}{h_{t}^{l}}$. First, the higher the distance $\frac{h_{t}^{h}}{h_{t}^{m}}$, the lower the income of the middle class relative to the mean, and therefore the more redistribution is required toward the middle-income class to form the club $\{h, m\}$. Second, a rise in $\frac{h_{t}^{m}}{h_{t}^{2}}$ yields an increase of the income of the middle class relative to the average and also increases the pressure in favor of higher redistribution. This result may appear somehow surprising, especially in light of the early political economy theory and conventional wisdom (see Alesina and Rodrik [1] , and Persson and Tabellini [35], who predict that redistribution will be higher the poorer the median voter is relative to the mean). However, in our scenario the underlying argument is straightforward. Let there be two economies 1 and 2 , and suppose first that $h_{1 t}^{h}=h_{2 t}^{h}$ and $h_{1 t}^{m}=h_{2 t}^{m}$, but $h_{1 t}^{l}=2 h_{2 t}^{l}$; that is, in the second economy, there is a higher wealth bias against the poor while at the same time the income of the middle class relative to the average is higher. This also means that the second economy is on average poorer than the first one which is itself more homogeneous than the former. As a consequence, in the second economy, more redistribution is required for the middle-income class to join the high-income class so that they can benefit from the same level of public good. Second, suppose now that $h_{1 t}^{l}=h_{2 t}^{l}$ and that $h_{1 t}^{h}=\frac{1}{2} h_{2 t}^{h}$ and $h_{1 t}^{m}=\frac{1}{2} h_{2 t}^{m}$, the second economy is now on average richer than the first one. Moreover, its 
middle-income class is richer relative to the average. What is important in this case to understand why $\widetilde{\tau}_{\{h, m\}}$ is higher in the second compared to the first economy is the fact that the absolute income gap between both the middle- and the high-income class is now higher in the second compared to the first economy. In both cases, the prospect of upward mobility underlying the middle-income class decision leads her to favor higher redistribution levels than advocated by the endogenous fiscal policy approach in the same range of inequality.

Corollary 1. The pressure for redistributive policies is not necessarily smaller in a rich (poor) economy where the income of the middle class is high relative to the average compared to the pressure that prevails in a poorer (richer) economy where the income of the middle class may be smaller than the average.

We now turn to Region $I I I$ depicted in Figure 4 and which corresponds to ranges of inequality where the political equilibrium is characterized as follows:

Proposition 3. When the income distribution is such that the middle-income class prefers to remain on its own rather than forming a club $\{m, l\}, \tau_{\{m\}}^{*}$ is the Condorcet winner which yields the equilibrium partition $P_{t}^{E}=(\{h\} ;\{m\} ;\{l\})$ as soon as the ex ante pattern of income distribution is such that $V_{t}^{m}\left(\{m\} ; \tau_{\{m\}}^{*}\right)>$ $V_{t}^{m}\left(\{h, m\} ; \tilde{\tau}_{\{h, m\}}\right)$ or $V_{t}^{l}\left(\{l\} ; \tau_{\{m\}}^{*}\right)>V_{t}^{l}\left(\{l\} ; \widetilde{\tau}_{\{h, m\}}\right)$ depending on whether $h^{m} / \bar{h} \gtreqless 1$.

Proof. See Appendix A

In Region $I I I$ where $\tilde{\tau}_{\{h, m\}}>\tau_{\{m\}}^{*}$, the equilibrium partition $(\{h\} ;\{m\} ;\{l\})$ is not necessarily the most preferred outcome for the middle-income class. Indeed, in that region there exist levels of inequality such that even though the middle-income class may most prefer a partition $(\{h, m\} ;\{l\})$ associated with a level of redistribution $\tilde{\tau}_{\{h, m\}}$, both agents of type $h$ and $l$ prefer a lower level of redistribution $\tau_{\{m\}}^{*}$ which can be defeated in pairwise comparison by no other level of redistribution (see the proof of Proposition 3 in Appendix A).

Corollary 2. Because of non-single peakedness, there exist in some range of inequality political equilibria where both the low-and the high-income classes agree on a similar level of redistribution which can not be defeated in binary contest. In other words, the pivotal voter does not always belong to the middle-income class. 
Moreover, this level of redistribution is smaller than the one most preferred by the middle-income class.

Intuitively, this result occurs for the following reason. The low-income class agrees with the high-income class to oppose high levels of redistribution because they want to prevent the middle-income class to form a club $\{h, m\}$ from which they are implicitly excluded. This scenario occurs in our framework when the low-income class can be better off by remaining on its own with a lower level of redistribution than required for the middle to join the highincome class. In other words, when high levels of redistribution lead the low-income class to lag further behind the other two income classes while they could catch up with a lower level of redistribution, they may oppose high rates of redistribution together with the high-income class. (See also Fernandez and Rogerson [20] who argue in favor of this kind of political outcomes, and Epple and Romano [18] and the so-called "ends against the middle" problem but in the latter case in a context of dual provision of education where an agent can consume either public or private education but not both, and where all agents who choose public school services obtain the same level of education.)

Recall that the equilibrium partition $(\{h\} ;\{m\} ;\{l\})$ in Region $I I I$ is associated with $\tau_{\{m\}}^{*}=\max \left\{0, \frac{1}{2}\left(1-\frac{h_{t}^{m}}{\bar{h}_{t}}\right)\right\}$. By definition, a decrease in $\frac{h_{t}^{h}}{h_{t}^{m}}$, respectively an increase in $\frac{h_{t}^{m}}{h_{t}^{2}}$, leads to a decline in $\tau_{\{m\}}^{*}$. At first sight, this corroborates the view that the richer the middle-income class relative to the average, the lower the pressure for redistribution. However, notice that this increase in $\frac{h_{t}^{m}}{\bar{h}_{t}}$ may have two sources. First, it may be the result of a middle-income class catching up with the high-income class. Second, it may be issued by the low-income class lagging further behind both the middle- and the high-income groups. Furthermore, both $\frac{h_{t}^{h}}{h_{t}^{m}}$ and $\frac{h_{t}^{m}}{h_{t}^{l}}$ may increase so that the Gini coefficient also increases $^{7}$, this will not necessarily lead to more redistribution if the rise in inequality deteriorates relatively more the situation of the low-income class relative to the middle-income class compared to the deterioration of the situation of the middle-income relative to the high-income class. This strengthens the argument of fiscal conservatism proposed by Saint Paul [37] who argues that the joint rise of inequality and fiscal conservatism that one could observe in the 1980s

\footnotetext{
7 Using the implicit function theorem, the iso-redistribution curve $\tau_{\{m\}}^{*}=\bar{\tau}$, is strictly increasing, convex in our plane and of slope: $\left.\frac{d\left(h^{m} / h^{l}\right)}{d\left(h^{h} / h^{m}\right)}\right|_{\tau_{\{m\}=\bar{\tau}}^{*}}=\left(\frac{h^{m}}{h^{l}}\right)^{2}$.
} 
and 1990s in industrialized countries such as the United States and the United Kingdom and in less developed countries such as Mexico and Argentina may be explained by the increase in inequality which disproportionately affected the bottom portion of income distribution.

Corollary 3. In a segmented economy, a rise in the wealth bias against the poor or even an increase in the Gini coefficient may yield a lower or similar equilibrium level of redistribution.

We shall now be interested in Region $I V$ displayed in Figure 4 where the following political equilibrium occurs

Proposition 4. When the ex ante pattern of income distribution is such that: (i) the level of redistribution required to form a club $\{h, m\}$ is higher than the level of redistribution required to form a club $\{m, l\}$; that is, $\widetilde{\tau}_{\{h, m\}}>\widetilde{\tau}_{\{m, l\}}$, and (ii) the middle-income class is better off in a club $\{m, l\}$ associated with either a level of redistribution $\tau_{\{m, l\}}^{*}$ or $\left(n h^{h}-(n+\beta) h^{m}\right) / \beta \bar{h}$ compared to both clubs $\{m\}$ and $\{h, m, l\}$ associated respectively with tax rates $\tau_{\{m\}}^{*}$ and $\widetilde{\tau}_{\{h, m, l\}}$, the equilibrium partition is $P_{t}^{E}=(\{h\} ;\{m, l\})$. The Condorcet winner is either $\tau_{\{m, l\}}^{*}$ or $\left(n h^{h}-(n+\beta) h^{m}\right) / \beta \bar{h}$ depending on whether $\tau_{\{m, l\}}^{*} \gtreqless\left(n h^{h}-(n+\beta) h^{m}\right) / \beta \bar{h}$, even though the middle-income class may be better off in that range of inequality in a club $\{h, m\}$ associated with a higher level of redistribution $\widetilde{\tau}_{\{h, m\}}$.

Proof. See Appendix A

Region $I V$ is located below the $45^{\circ}$-line; that is, the low- and the middle-income classes are now more homogeneous and there is a strong wealth bias in favor of the high-income class compared to Region $I I$. Whenever there is a rise in $\frac{h_{t}^{h}}{h_{t}^{m}}$ and/or $\frac{h_{t}^{m}}{h_{t}^{2}}$, redistribution increases although one can now easily understand that when the income share of the low-income class decreases relative to the others, the pressure for redistribution increases for the club $\{m, l\}$ to become feasible. (Corollary 1 therefore also applies in Region $I V$.)

"Ends against the middle" situations as described in Corollary 2 also arise in Region $I V$. Indeed, it is not necessarily the case that the partition $(\{h\} ;\{m, l\})$ corresponds to the middle-income agent's most preferred outcome of the political process. Even though the middle-income class might be best off within a club $\{h, m\}$ associated with the tax 
rate $\widetilde{\tau}_{\{h, m\}}$, the club $\{m, l\}$ associated with a level of redistribution $\tau_{\{m, l\}}^{*}$ or $\left(n h^{h}-(n+\beta) h^{m}\right) / \beta \bar{h}$ is preferred by both the low- and the high-income classes and therefore may block the partition $(\{h, m\} ;\{l\})$. It is also worth noticing that when $\left(n h^{h}-(n+\beta) h^{m}\right) / \beta \bar{h}<\tau_{\{m, l\}}^{*}$, then the club $\{m, l\}$ emerges with a level of redistribution which does not provide the maximum level of quasi-public resources which could be available to agents of type $m$, and $l$. Indeed, in that range of inequality, the partition $(\{h\} ;\{m, l\})$ associated with $\tau_{\{m, l\}}^{*}$ is blocked by the coalition $\{h, m\}$. Further, the emergence of a political coalition between the low- and the high-income classes does not necessarily require that the most preferred tax rate of the middle-income class involves high deadweight costs.

Finally, we can provide the following:

Proposition 5. All alternative ex ante income distributions which do not enter the above 4 propositions yield

\section{Condorcet cycles.}

The range of inequality where there is no political equilibrium under pure majority rule is displayed in Region $V$ of Figure 4. It is well-known that in this case there are strong incentives for strategic manipulation, either of the agenda itself or of the preferences revealed in the voting process. We can think of two ways to solve this problem. First, imposing additional institutional structure on the political process can give rise to a well-defined equilibrium (see for instance, Chapter 2 of Persson and Tabellini's reference book [36] ). Second, another voting rule could be chosen, namely the Borda rule (see for instance Moulin [31] ). However, at this stage, we leave this issue unexplored focusing on the likelihood that Condorcet cycles may arise in some key range of inequality.

We now analyze levels of redistribution across the different regions and provide a complementary explanation as to why government transfers may differ so greatly between countries. Let us consider three economies, $E_{I I}, E_{I I I}$, and $E_{I V}$ characterized by levels of relative inequality as is displayed in Figure 5. Keep in mind that we have no information about whether $E_{I I I}$ characterizes a richer or a poorer economy compared to $E_{I I}$. In this figure, we first 


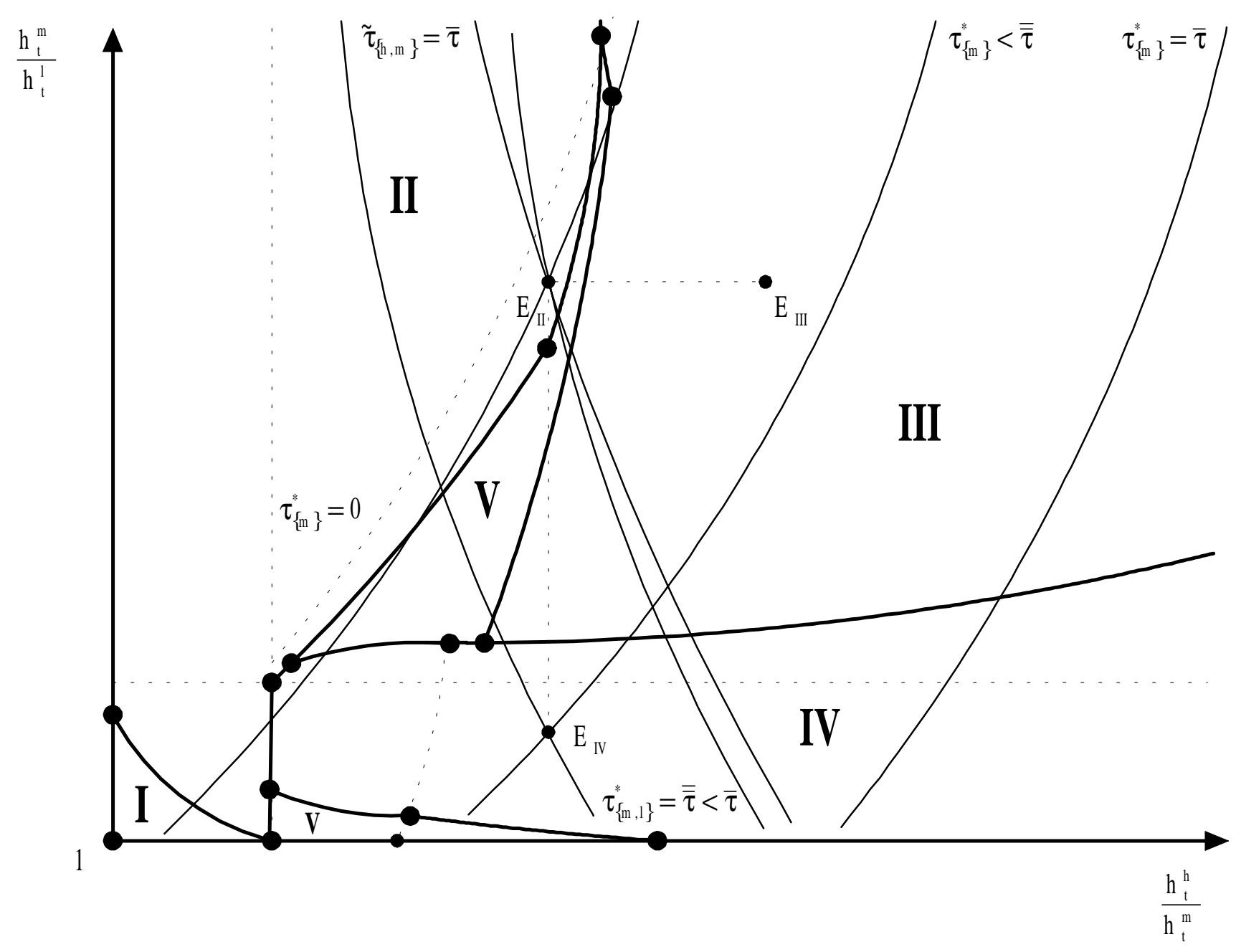

Figure 5: Equilibrium partition regions in the plane $\left(\frac{h_{t}^{h}}{h_{t}^{m}}, \frac{h_{t}^{m}}{h_{t}^{i}}\right)$, and iso-redistribution curves. 
plot iso-redistribution curves such that for instance $\tilde{\tau}_{\{h, m\}}=\bar{\tau}$ and $\tau_{\{m\}}^{*}=\bar{\tau}$, and such that $\tau_{\{m, l\}}^{*}=\overline{\bar{\tau}}<\bar{\tau}^{8}$. Notice first that $E_{I I}\left(E_{I I I}\right)$ is located in region $I I(I I I)$ where the corresponding equilibrium partition is $(\{h, m\} ;\{l\})$ $((\{h\} ;\{m\} ;\{l\}))$. Second, the position of $E_{I I}$ and $E_{I I I}$ in the plane, is such that the middle-income class in $E_{I I I}$ is poorer relative to the average compared to the middle-income class in $E_{I I}$. However, notice that in both economies $E_{I I}$ and $E_{I I I}, \tilde{\tau}_{\{h, m\}}>\tau_{\{m\}}^{*}$, it is then clear that the equilibrium tax rate $\left.\tau_{\{m\}}^{*}\right|_{E_{I I I}}$ which defeats in binary contest all alternative redistribution patterns in region $I I I$ is lower than $\left.\tilde{\tau}_{\{h, m\}}\right|_{E_{I I}}$ which is the Condorcet winner in region $I I$. Finally, $E_{I V}$ located in region $I V$ is also an economy characterized by a middle-income class which is poorer relative to the mean than it is in the first economy $\left(E_{I I}\right)$. Provided that $E_{I I}$ is located in the plane where the pattern of income distribution is such that $\widetilde{\tau}_{\{h, m\}}>\tau_{\{m, l\}}^{*}$, it is also straightforward given the slopes of the iso-redistribution curves, that $\left.\tau_{\{m, l\}}^{*}\right|_{E_{I V}}<\left.\widetilde{\tau}_{\{h, m\}}\right|_{E_{I I}}$. Finally, whatever the level of inequality, $\tau_{\{m, l\}}^{*}>\tau_{\{m\}}^{*}$, therefore, the level of redistribution is also lower in $E_{I I I}$ compared to $E_{I V}$. The link between the pressure for corrective policy action in favor of more equality and income distribution is nothing but automatic. High levels of redistribution are not always associated with large inequalities yielding multiple politico-economic regimes. Following Bénabou [7] although relying on another mechanism, we can therefore also provide the following:

Corollary 4. More unequal and segmented economies may redistribute less, not more.

\subsection{A First Step Toward Uncovering Multiple Politico-Economic Regimes in the Data}

Bénabou [6] provides an insightful review and discussion of the empirical findings about the relationship between inequality and the share of transfers. In fact, no empirical regularity of the kind argued by the endogenous fiscal policy approach of Alesina and Rodrik [1] and Persson and Tabellini [35], among others, can be inferred from the traditional regressions across a worldwide set of countries. Perotti [33] and [34], for instance, carefully tests the relationship between inequality and the share of transfers in GDP as proxied by different types of government expenditure within

\footnotetext{
$8 \quad$ Let us here consider the iso-redistribution curves $\tilde{\tau}_{\{h, m\}}=\bar{\tau}$ and $\tau_{\{m, l\}}^{*}=\bar{\tau}$. It is easily shown that both are strictly decreasing and convex in our plane. Moreover, we also have $-\left.\frac{d\left(h^{m} / h^{l}\right)}{d\left(h^{h} / h^{m}\right)}\right|_{\tau_{\{m, l\}=\bar{\tau}}^{*}}<-\left.\frac{d\left(h^{m} / h^{l}\right)}{d\left(h^{h} / h^{m}\right)}\right|_{\tilde{\tau}_{\{h, m\}=\bar{\tau}}}$.
} 
a cross-section of countries where either the share of the third quintile or the combined share of the third and fourth quintiles, is used as a proxy for the income of the median voter relative to the average. The relationship is found to be positive in Perotti [33], respectively negative in Perotti [34], but in most cases, it is not significant even when controlling for democracy. In our model, when the income of the middle class increases relative to the mean, redistribution increases in Regions $I I$ and $I V$ but decreases in Region $I I I$. As a consequence, this relationship is expected to be non linear because of the existence of multiple politico-economic regimes as discussed above. Patterns of redistribution are richer than that proposed by the standard political economy models where in equilibrium the median income on one side and the level of expenditure and taxation on the other are negatively related. The empirics of inequality and redistribution should definitely take into consideration that distributional tensions act in many other subtle ways leading patterns of redistribution to be highly non linear. Using a more recent and accurate dataset measuring income inequality and collected by Deininger and Squire [13] , Figini [22] also finds that countries with high inequality (here measured by the Gini coefficient) are the ones that on average although not significantly, redistribute less in terms of different types of redistribution measures among which is the ratio of government expenditure to GDP. Still, adding a squared term to his cross-country regression, Figini finds a significant U-shaped effect of income inequality on the shares of tax revenues and government expenditure in GDP therefore corroborating the non monotonic relationship predicted above and by the model in Bénabou [7] .

However, notice that in our model, the equilibrium level of redistribution does not only depend on the income of the middle class relative to the average. Instead, we argue that a synthetic measure such as the Gini coefficient or the percentages of income accruing to the third quintile and to the sum of the third and fourth quintile, is likely to miss important mechanisms through which the balance of power in the political system is affected by the pattern of income distribution. Our model rather suggests the relative income gap between the different income classes as the relevant variables to be used to explain why the share of government expenditure in GDP differs so greatly between countries. Lindert [28], focusing on a subset of OECD countries over the period 1960-1981, emphasizes and highlights the role of social affinity and therefore of the upper and lower income gaps as a two key dimension of the income distribution 
to understand the great variation of government transfers across countries.

Let us start by discussing the information provided in Table 1 for a subset of OECD countries. In this table, countries are ranked according to their share of total government expenditure in GDP. We also provide the ratio of education expenditure to GDP whose correlation coefficient with the ratio of total government expenditure to GDP is equal to 0.77. Two synthetic measures of the income distribution, the Gini coefficient and more naturally the sum of the share of the third and fourth quintiles which captures the notion of middle class, are also available. Given this information, we then rank countries according to what is expected from the endogenous fiscal policy approach; that is, more unequal countries are expected to redistribute less, and on the other hand, the lower the middle class income, the higher the share of transfers. First, notice that the correlation coefficient between the ratio of government expenditure to GDP and the Gini coefficient, respectively the share of the third and fourth quintiles, is only equal to 0.25 , respectively -0.31 . Second, looking at this table and especially focusing on the share of the third and fourth quintiles, we are clearly back to the puzzle raised by Bénabou [7] which is also our main focus in this paper. Scandinavian countries are on average both more equal and more redistributive than the United States or even compared to European countries like Germany, Spain, or the United Kingdom.

Our approach though differs greatly from Bénabou [7] . Following Lindert [28], it rather stresses a two key dimension of the income distribution which emphasizes the role of the relative social proximity or affinity between the different income classes in understanding the various patterns of public spending across countries. In the figure available in Table1, are depicted data available in the last two columns of Table 1. We then add our theoretical apparatus as displayed in Figure 5. The existence of multiple politico-economic regimes allows us to account for the differences in total public spending as a percentage of GDP across our sample in a more accurate fashion compared to what is implied by models where (high) inequality measured by the gap between the median and the mean income is monotonically associated with higher redistribution.

First, in accordance with the fiscal policy approach and our model, moving North-West in Region $I I I$ yields an increase in the income of the middle class relative to the average which in turn translates into a decrease in government 


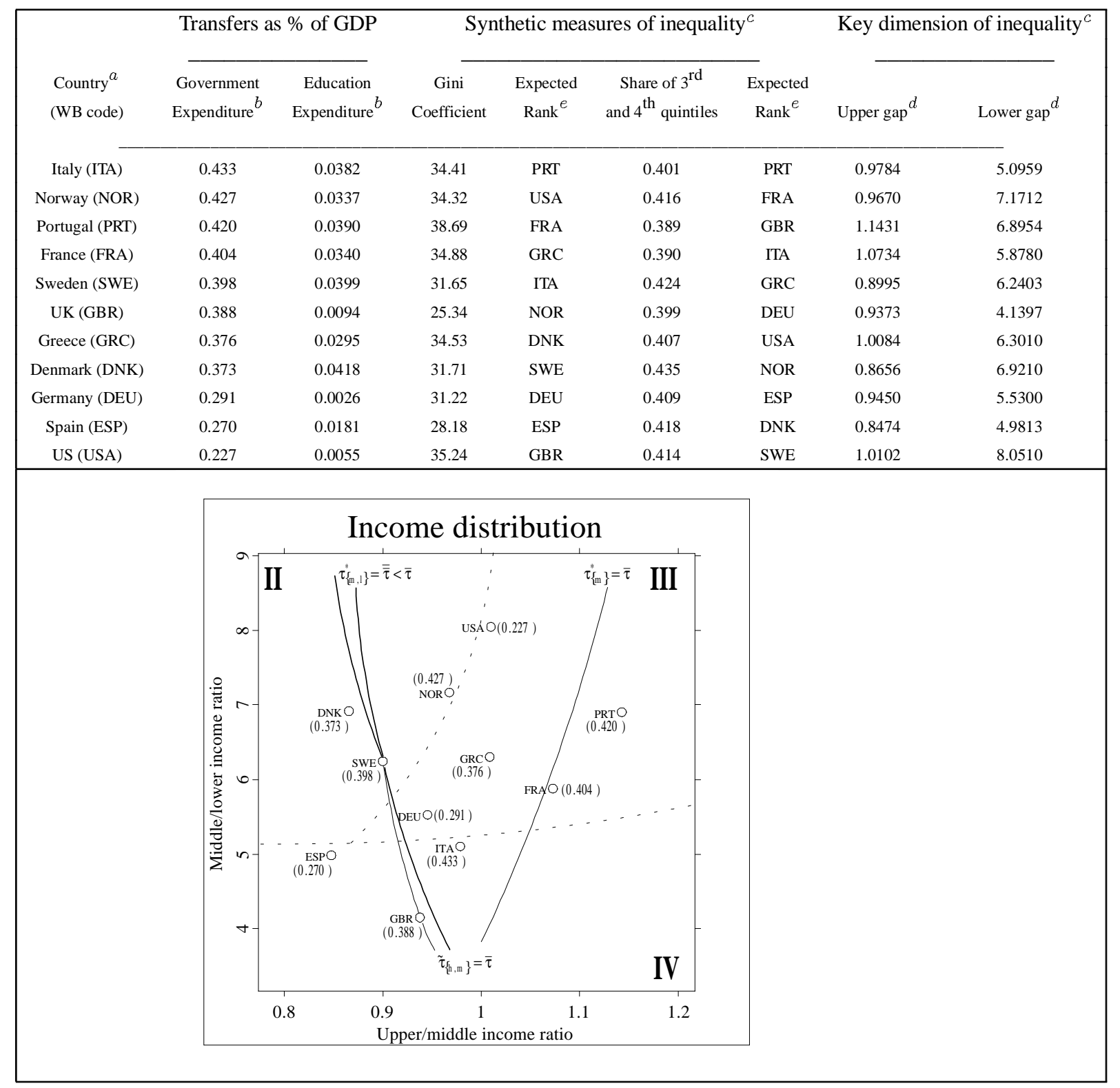

Table 1: Shares of total government and education expenditures in GDP and measures of inequality.

Notes:

- a. Countries are ranked according to their shares of total government expenditure in GDP.

- b. Average over all available observations between 1970 and 1988 of total expenditure and lending minus repayments and of expenditure on education as shares of GDP, issued by the IMF Government Financial Statistics. Source: Easterly and Rebelo (1993).

- c. Average over all available observations between 1960 and 1988. Source: "high quality" dataset collected by Deininger and Squire (1996).

- d. The upper, respectively lower, gap is the ratio of the share of income for the top quintile to that for the sum of the third and fourth quintiles, respectively the comparable measure between the sum of the third and fourth quintiles to that of the bottom quintile. Notice that the correlation coefficient between the third quintile and the sum of the third and fourth quintile is 0.93 .

- e. Ranking of countries according to what is expected from the endogenous fiscal policy approach; that is, government transfers increase with inequality as measured by the Gini coefficient and are negatively related with the income share of the sum of the third and fourth quintiles which is used to proxy the gap between the median and the mean income. 
redistribution. This result is also in accordance with the ranking of those countries which belong to Region $I I I$ in the figure displayed in Table 1 in terms of their share of the third and fourth quintiles. Second, if the three Scandinavian countries indeed belong to a different regime as defined in Region $I I$, then our model also predicts that even though being more equal, they can be expected to redistribute more compared to the United States and those countries which are relatively close to the frontier between Region $I I$ and Region $I I I$, namely Germany and Greece. Also in accordance with the prediction of our model is the increase in government transfers which is expected in Region $I I$ while moving along the North-East direction. Finally, the last regime located in Region $I V$ also fits the prediction of our model. Thus, as in Region $I I$, Italy is expected to redistribute more than the United Kingdom which must itself redistribute more than Spain. On the other hand, the levels of government spending which characterize these countries compared to others in Region $I I$ and $I I I$ are also in accordance with the above comparative static analysis across regions.

In Table 2, we provide regressions across a set of 42 countries of different types of shares of public expenditures in GDP on variables reflecting the relative upper gap measured by the high class income (top quintile) to the middle class income (third quintile) ratio, and the relative lower gap measured by middle class income and the low class income (bottom quintile) ratio. We initially specify a non linear relationship (see columns (1), (2), and (3)) up to the third degree, and only show the best nested model according to the LM-test proposed by Breusch and Pagan [10] within the more general specification. Notice first that social security and welfare is the type of tranfers for which the relationship between our two key dimension of the income distribution and redistribution ends up with the best fit $\left(R^{2}=0.63\right)$. The underlying model clearly and significantly exhibits non linearities where the first dimension (upper gap), respectively the second dimension (lower gap) as depicted in the figure below Table 2, is U-shaped, respectively inverted U-shaped. These non linearities are also siginificantly present when education expenditures are considered although the fit of the regression is much lower (the p-value associated with the F-test indicates that this regression is only significant at the 10 percent level). Second, recall on the one hand that social security and welfare is the only type of expenditure for which Perotti [34] is able to find a significant negative partial association with the middle class income measured by the combined share of the third and fourth quintiles. On the other hand, Figini [22] 


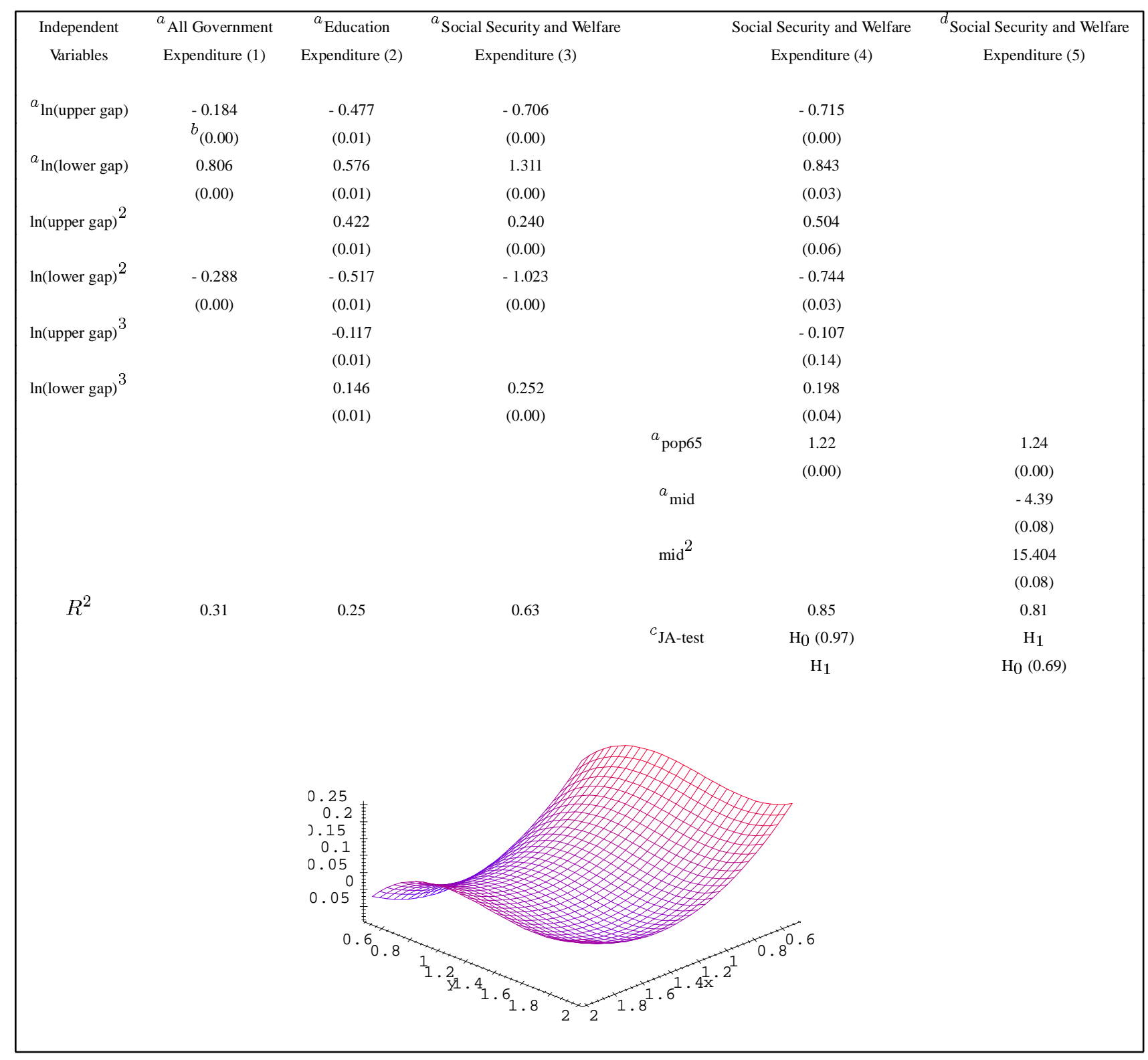

Table 2: The income distribution as a determinant of different types of public expenditures as percentages of GDP, and surface of the regression as estimated in Column (3) - along the $\mathrm{x}$-axis, respectively the $\mathrm{y}$-axis, is the $\ln$ (upper gap), respectively the $\ln ($ lower gap).

Notes:

- a. The dependent variables are different types of government expenditures as percentages to GDP (source: Easterly and Rebelo (1993)). Measures of inequality are as defined in Table 1 except that the middle class income is measured by mid, the share of the third quintile (source: the "high quality" data set collected by Deininger and Squire (1996)). pop65 is the average share of population over sixty-five years of age over the period 1970-1985 (source: Barro and Lee (1993)). The number of available observations amounts to 42 .

- b. p-values, i.e. the marginal significance level of a two-tailed test of the hypothesis that the coefficient is equal to zero, are in parentheses under coefficient estimates. - c. The JA-test performs a non nested specification test of both models in Columns (4) and (5). The p-values in parentheses give the probability of being wrong when rejecting the model specified under the null.

- d. The model in Column (5) is estimated with an intercept (not shown). 
finds a U-shaped relationship between inequality measured by the Gini coefficient and redistribution proxied by the percentage of all government expenditure to GDP. Following both authors, we propose to formally test our competing approach versus the endogenous fiscal approach. Results are available in columns (4) and (5) in Table 2 where we add as in Perotti [34] an important demographic variable, namely the share of population over sixty-five years of age. It indeed significantly increases the overall fit of the regression compared to result in column (3). Interesting is that the model initially aimed at describing the fiscal policy approach and therefore based on the share of the third quintile is better characterized by a quadratic relationship as already found by Figini [22] . Indeed, a LM-test rejects the null hypothesis in favor of a linear and monotonic relationship between the middle class income and the share of social security and welfare expenditure in GDP. Finally, we turn to testing between our model estimated in column (4) and the quadratic form estimated in column (5) that are non nested models. We apply a JA-test (see Fisher and Mc Aleer [24] ) whose results are available in Table 2 where the p-values given in parentheses give the probability of being wrong when rejecting the model specified under the null. Obviously, none of both models can be rejected. Nevertheless, the probability of being wrong when rejecting the model specified in column (4); that is the social affinity hypothesis, is much higher compared to that when rejecting the model in column (5) therefore supporting both our model and results found by Lindert [28] who also highlights the role of the income distribution measured by similar interquintile income ratios in understanding the great dispersion of public spendings across a subset of OECD countries.

This first step toward uncovering multiple politico-economic regimes in the data suggests that the balance of power which drives social transfers is more intricate than that proposed by standard political economy models where the level of taxation is expected to be negatively and monotonically associated with the income gap between the median voter relative to the average. The level of the middle class income relative to both the highest and the lowest class incomes appears to be a key determinant to understand why public transfers differ so greatly across countries, and more specifically why more unequal economies may redistribute less, not more in some range of inequality. Because of contemporary data and sample size problems, the above empirical evidence can certainly not be considered as the end of the story. However, it is encouraging and, at least partially, supports the kind of distributional tensions and the 
existence of multiple politico-economic regimes of the kind identified and discussed above.

\section{Dynamics}

We now concentrate on how inequality and redistribution influence the dynamic evolution of income distribution through time, human capital accumulation, and growth. We also provide a scenario which illustrates the forces setting the limits of government transfers. The evolution of the economy is characterized by the dynamics of $\frac{h_{t}^{h}}{h_{t}^{m}}$ and $\frac{h_{t}^{m}}{h_{t}^{h}}$. Recall that at time $t$, the income distribution not only determines the after-tax equilibrium, but also determines next period ex ante distribution of human capital stocks. As the pattern of income distribution changes over time, the political equilibrium shifts endogenously along the transitional path ${ }^{9}$. Similarly to Durlauf [15] , intergenerational group formation implies that income trajectories may be very different depending on the initial pattern of income distribution leading to the existence of multiple steady states. In our simple deterministic framework, we characterize intergenerational mobility along the transitional path. For instance, the evolving pattern of income distribution from Region $I V$ to Region $I I$ leads the middle-income class to experience upward mobility. It becomes homogeneous enough to the high-income class so that, in this range of inequality, there exists a political outcome which allows it to benefit from better local interaction in the community $\{h, m\}$.

More specifically, the dynamics of income inequality across agents of type $i$ and $k$ where $h_{t}^{i}>h_{t}^{k}$, who belong respectively to a club $S_{j t}$ and $S_{j^{\prime} t}$ at equilibrium, depend on the combination of two effects. Let us consider the following expression:

$$
\frac{h_{j, t+1}^{i}}{h_{j^{\prime}, t+1}^{k}}=\left(\frac{h_{t}^{i}}{h_{t}^{k}}\right)^{(1-\alpha-\beta)}\left(\left(\frac{\sum_{z \in S_{j t}}\left(h_{t}^{z}+\tau \bar{h}_{t}\right)}{n_{j t}} \cdot \frac{n_{j^{\prime} t}}{\sum_{z^{\prime} \in S_{j^{\prime} t}}\left(h_{t}^{z^{\prime}}+\tau \bar{h}_{t}\right)}\right)\left(\frac{n_{j^{\prime} t}+\beta}{n_{j^{\prime} t}} \cdot \frac{n_{j t}}{n_{j t}+\beta}\right)\right)^{\beta}
$$

The first effect is the traditional income convergence result due to diminishing returns in $G_{j t}$ and $h_{t}^{i}$, as emphasized by Tamura [41] , and Glomm and Ravikumar [27] , among others. Second, the neighborhood effects associated with the equilibrium partition which emerges also influence income dynamics in three specific ways captured in the second

9 Remind that, due to a limited bequest motive, our framework is not a dynamic game in which agents would care about future political outcomes. See for instance Saint Paul [37] for a dynamical voting game. 
term of Equation (12): (i) the per capita posttax income ratio between $S_{j t}$ and $S_{j^{\prime} t}$ forces divergence, (ii) but this ratio is negatively related to $\tau$; that is, redistribution drives convergence. This reflects the effect of redistribution which brings about more equality of opportunity to save and invest a greater amount of income into education or to benefit from higher levels of educational services and local externalities. (iii) An ambiguous size effect which yields convergence, respectively divergence, when the higher-income community is smaller, respectively larger, compared to the lower-income community.

The underlying global dynamics of the economy depend on the interplay between these two effects. Given Propositions 1 to 5 and information about the evolution of income dynamics provided in Appendix $\mathrm{C}$, we can construct the phase diagram as displayed in Figure 6.

As can be seen from Figure 6, the long run behavior of the income distribution is such that there exist two steady states. They describe either a situation of equality and integration or segregation with persistent inequality. Denoting $\Pi$ by

$$
\Pi=\ln \left(\frac{2 n+\beta}{2 n}\right) / \ln \left(\frac{2(n+\beta)}{2 n+\beta}\right)>1
$$

we can provide the following:

Proposition 6. If and only if $\beta / \alpha \geq \Pi$; that is, the isokine $y_{t+1}=\left.y_{t}\right|_{\tilde{\tau}_{\{h, m\}}=0}$ is always located above the locus $\tilde{\tau}_{\{h, m, l\}}=0$, there exist two steady states:

1/ One called the integrated equilibrium (IE) and characterized by a completely homogeneous population which belongs to the same community. It is a globally stable steady-state within Region I.

2/ The second, called the segregated equilibrium (SE), is such that the initially high-and middle-income classes consist of an homogeneous community while the low-income class remains isolated. In this case, there is persistent inequality. It is a locally stable steady state within Region II.

Otherwise, there is a unique steady state which is the integrated equilibrium.

Proof. See Appendix C

Income inequalities are not necessarily completely eliminated in the long run. The multiple steady states situation 


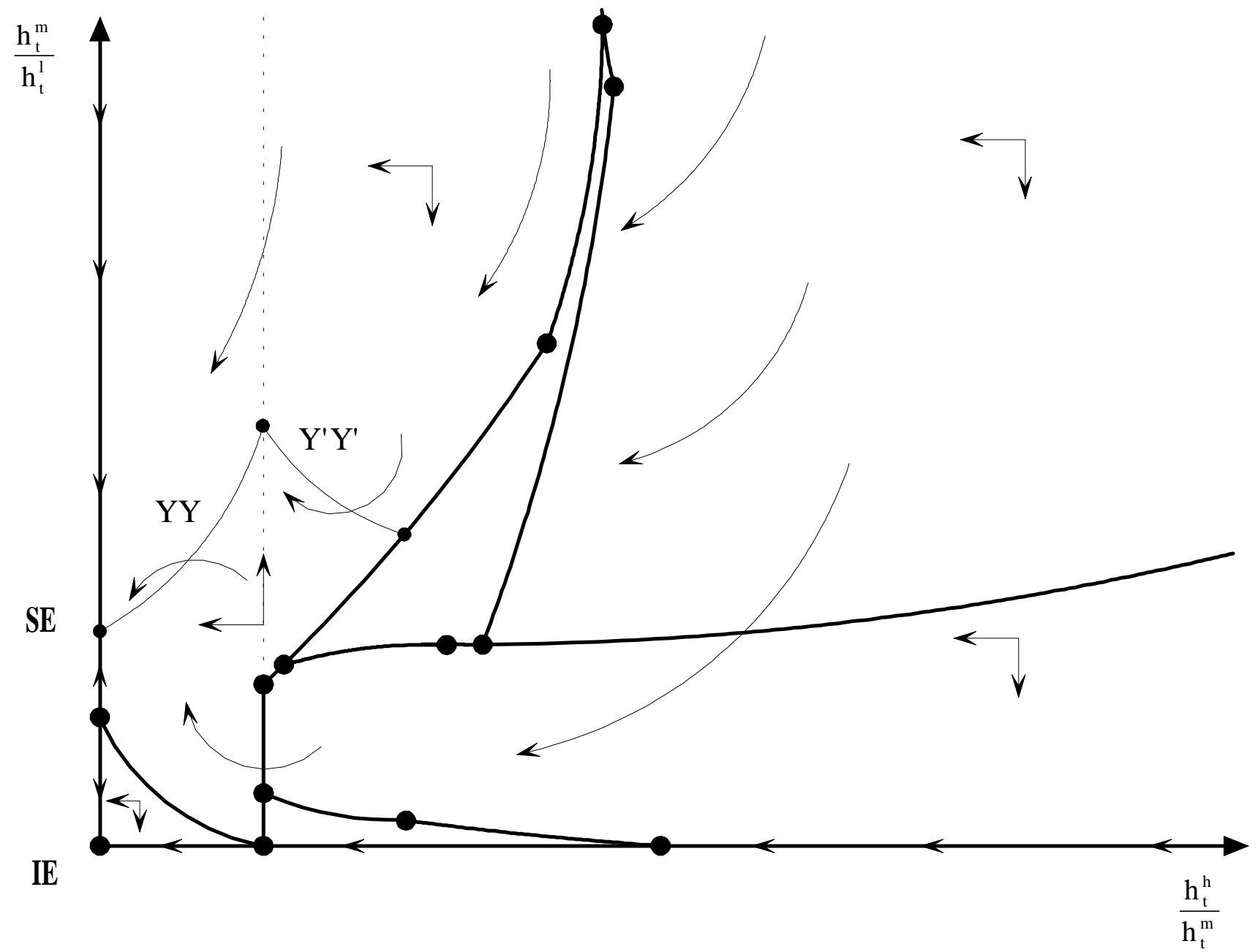

Figure 6: Inequality dynamics. SE, respectively IE, denotes the segregated, respectively the integrated, equilibrium. $Y Y\left(Y^{\prime} Y^{\prime}\right)$ corresponds to the isokine $y_{t+1}=\left.y_{t}\right|_{\tilde{\tau}_{\{h, m\}}=0}\left(y_{t+1}=\left.y_{t}\right|_{\tilde{\tau}_{\{h, m\}}>0}\right)$. 
occurs when the local versus global externalities ratio $(\beta / \alpha)$ reaches a threshold level as defined in Equation (13). Otherwise, if $\beta / \alpha<\Pi$, then the economy-wide spillovers are strong enough and inequality vanishes in the long run. (See also Durlauf [14] who stresses how much the relative size of the local spillovers is important to obtain multiple steady states.)

The integrated equilibrium is characterized by a completely homogeneous population where individual incomes are all equalized. When the three income classes interact in the grand coalition, this equilibrium partition remains forever; that is, as soon as the economy is in that range of inequality defined by Region $I$, the positive feedbacks spread uniformly over the whole population with no need for redistributive policies. On the contrary, at the segregated equilibrium, inequality is persistent. While the high- and the middle-income classes benefit from the same level of local externalities and are similarly endowed, the poorer individuals remain stuck into a wealth trap where they are not able to benefit from the local linkage available in the richer community. The long-run inequality is characterized by a permanent income gap between the rich community and the low-income class equal to $\left(\frac{2(n+\beta)}{2 n+\beta}\right)^{\beta / \alpha}$. Notice that this steady state corresponds to a situation where no redistribution is now preferred by a majority of the population. As a consequence, there is still room for a central planner to influence this locally stable steady state through one-shot redistribution policies until the pattern of income distribution reaches Region $I$.

Finally, in a segregated equilibrium polarization leads the above size effect to lower growth compared to the integrated equilibrium. Moreover, as the specified local public good technology is characterized by perfect substitutability between the individual investment effort and that the economy-wide knowledge spillovers are simply an arithmetic average, steady-state heterogeneity reduces growth ${ }^{10}$.

Proposition 7. The integrated equilibrium has higher growth than the segregated one.

\section{Proof. See Appendix C}

Clearly, this proposition also stresses the long-run inefficiency of social polarization as all dynasties are hurt by a 10 See Bénabou [5] who studies the effect of stratification when the degree of complementarity between individuals' human capital stock varies
depending on both local and global interactions. 
slower aggregate human capital accumulation.

We now turn to the description of the global dynamics of our framework. Then, we address the issue of the impact of the introduction of redistribution on income trajectories and growth.

Let us consider an economy starting in Region $I I I$. From Proposition 3, inequality is relatively high such that each income class forms its own community and the associated equilibrium level of redistribution is $\tau_{\{m\}}^{*}$. As displayed in Figure 6, local and global spillovers lead both ratios $\frac{h_{t}^{h}}{h_{t}^{m}}$ and $\frac{h_{t}^{m}}{h_{t}^{h}}$ to decrease. Over a finite time, the community structure of the economy evolves towards a new equilibrium partition. Depending on the initial income distribution, the economy may enter in Region $I I, I V$, or $V$. Suppose that the new regime is Region $I V$; that is, the low-income dynasty experiences an upward mobility movement as it reaches a posttax income high enough to interact with agents of type $m$ in the community $\{m, l\}$. The global effect on the dynamics of income is that the three income classes become more and more homogeneous. Three possibilities can then arise. First, the low- and middle-income classes become similar and the grand community is now preferred by a majority. Second the economy enters in the Condorcet cycle region. Third, the economy reaches Region $I I$ as the middle-income class catches up to the rich agents and is now accepted in their club while the poorer individuals remain in a community with only people of the same kind. In this case, the middle-income dynasty is upward moving while the poor dynasty experiences a downward social mobility. Suppose dynamics are such that the economy enters in Region $I I$. The ratio $\frac{h_{t}^{m}}{h_{t}^{l}}$ increases because the local interaction in community $\{h, m\}$ combines two divergence forces: a richer club and a bigger size than in the club $\{l\}$. After a finite time, two transitions are possible. Either social polarization is enhanced and the economy converges to the segregated equilibrium, or, despite increasing $\frac{h_{t}^{m}}{h_{t}^{2}}$, the low-income class is rich enough to be accepted in a club $\{h, m, l\}$ and the economy enters into Region $I$. In this case, the economy evolves toward the integrated equilibrium.

Global dynamics of inequality are characterized by history-dependent steady states. The initial political equilibrium which emerges, determines the human capital accumulation of all subsequent generations. Thus, when the initial pattern of income distribution exhibits a strong wealth bias against the poor (extreme North West of Figure 6), the economy ends up polarized. On the opposite, when the initial pattern of income distribution is such that the low- and middle-income 
classes are homogeneous but very far from the high-income-class (extreme South East of Figure 6), the economy ends up integrated.

It is also worth noticing the crucial feature of the Condorcet regions: many economies starting from Region $I I I$ and $I V$ can be confronted with these political conflicts along their transitional path. Nevertheless the chance to achieve the integrated equilibrium decreases with the relative importance of local externalities. Indeed, there exists a critical value of $\beta / \alpha$ denoted $\Gamma$ with

$$
\Gamma=\ln \left(\frac{\frac{2 n+\beta}{n}}{1+\left(\frac{n+\beta}{n}\right)^{(1-\alpha-\beta)}}\right)^{\frac{1}{\alpha}} / \ln \left(1+\left(\frac{n}{\beta}\right)^{-1}\right)>\Pi
$$

such that,

Proposition 8. When $\frac{\beta}{\alpha} \geq \Gamma$, whatever the political outcome that would emerge in Region $V$, only economies with an initial pattern of income distribution located in Region I converge towards the integrated equilibrium.

\section{Proof. See Appendix C}

Thus, when the local determinants of education are relatively important, whatever the intergenerational mobility which occurs along the transitional path, the only possibility to obtain long run equality is that everybody initially benefits from the same neighborhood spillover effects. At the extreme when $\alpha=0$, any initial pattern of income distribution leading to separation of agents results in an income gap between a rich club with size $2 n$ and the poor class which nevertheless keeps growing. Further, despite the fact that the area in which $(\{h, m\} ;\{l\})$ emerges as the equilibrium partition spreads out, the introduction of redistribution enhances the probability to reach the integrated equilibrium at the steady state. We can indeed provide the following

Proposition 9. Introducing redistribution increases the number of candidates for the integrated equilibrium.

Moreover, redistribution favors growth.

Proof. See the above discussion

Compared to the case without any fiscal policy, redistribution enhances aggregate human capital accumulation 
despite deadweight losses. This result relies on the fact that ex ante income heterogeneity is reduced leading human capital technology to be more efficient.

\section{Conclusion}

In a world where the level of educational services available to an individual strongly depends on her capacity or ability to invest into education but also on the incentive to do so through the affiliational community she may belong to, the conflicts of interest which arise may be more complex than advocated in the conventional political economy literature. More specifically, we abandon the stylized median voter in favor of more nuanced pressure-group reasoning. Depending on the social break-up, our main result is that the pressure for redistributive taxation is highly non linear and international empirical evidence tends to support our argument. Moreover, it is not necessarily higher in more unequal and segmented economies, therefore limiting government spending and redistributive programs in some range of relatively high inequality.

The present community structure strongly depends on historical backgrounds and shocks which can explain why across a worldwide set of nations, we observe such a highly non-linear and uneven redistributive pattern. Moreover, most democracies can be expected to face ranges of inequality where voting cycles may occur. Additional structure should be imposed in our model by restricting the institutions which govern how policy decisions are made. At this stage, this issue is left unexplored and postponed to a future research agenda.

\section{Appendix A. Proofs of Propositions 1 through 5}

The voting rule which solves the collective decision problem is issued by the Condorcet method where the political outcome of the vote is the candidate which defeats every other candidate in pairwise comparison. Each candidate is associated with an equilibrium partition which may differ according to the ex ante pattern of income distribution. Recall first that the high-income class has no incentive to redistribute so that we always have for agents of type $h$ : $0 \succeq \tau_{\{m\}}^{*} \succ \tau_{\{m, l\}}^{*} \succ \tau_{\{l\}}^{*} \succ \frac{1}{2}$. Second, our assumption about the distortions associated with the redistributive 
policy leads a level of redistribution $\tau \geq 1 / 2$ to be always preferred in binary contest by all the other candidates which are:

$$
\tilde{\tau}_{\{h, m, l\}}, \tilde{\tau}_{\{h, m\}}, \tilde{\tau}_{\{m, l\}}, 0, \tau_{\{m\}}^{*}, \tau_{\{m, l\}}^{*}, \tau_{\{l\}}^{*}
$$

Proof of Proposition 1 First, when the ex ante pattern of inequality is such that $n\left(h_{t}^{h}+h_{t}^{m}\right)-(2 n+\beta) h_{t}^{l}<0$, all income classes are able to positively contribute to the provision of the public good in the club $\{h, m, l\}$ for any level of redistribution. Hence, for any tax rate $\tau \geq 0$, the club $\{h, m, l\}$ provides the highest level of public good to all income classes. Notice that in that range of inequality, $\tau_{\{h, m, l\}}^{*}=\widetilde{\tau}_{\{h, m, l\}}=0$, then the level of public good available in $\{h, m, l\}$ is maximum when no distortionary redistribution occurs. As a consequence, the equilibrium partition is $P_{t}^{I}=(\{h, m, l\})$ and all income classes vote in favor of no redistribution. Second, notice that when $h_{t}^{m}=h_{t}^{l}$ the low- and the middle-income classes are similarly endowed ex ante. It implies that $\widetilde{\tau}_{\{h, m, l\}}=\widetilde{\tau}_{\{h, m\}} \geq 0$ and $\tau_{\{m, l\}}^{*}=\tau_{\{m\}}^{*}=\tau_{\{l\}}^{*}$. As long as $V_{t}^{i}\left(\{h, m, l\} ; \widetilde{\tau}_{\{h, m, l\}}\right) \geq V_{t}^{i}\left(\{m, l\} ; \tau_{\{m, l\}}^{*}\right)$ for $i=m, l$ the political outcome of the vote is $\widetilde{\tau}_{\{h, m, l\}}$ associated with the equilibrium partition $P_{t}^{I}=(\{h, m, l\})$.

In order to prove the four other propositions, we need some information provided in Lemma 1.

Lemma 1 When the initial pattern of income distribution is such that $n\left(h_{t}^{h}+h_{t}^{m}\right)-(2 n+\beta) h_{t}^{l} \geq 0$ and $h^{m}>h^{l}$, $\tilde{\tau}_{\{h, m\}}$ is most preferred by both the middle- and the high-income classes compared to $\tilde{\tau}_{\{h, m, l\}}$. Hence, in that range of inequality, $\tilde{\tau}_{\{h, m, l\}}$ cannot be a Condorcet winner. It can just be a candidate which yields Condorcet cycles. Moreover, when the ex ante pattern of inequality is such that $n h_{t}^{h}-(n+\beta) h_{t}^{m} \geq 0$ and $n h_{t}^{m}-(n+\beta) h_{t}^{l} \geq 0, \tilde{\tau}_{\{h, m, l\}}$ is not a relevant candidate any more because it implies that $\widetilde{\tau}_{\{h, m, l\}}>1$.

Proof. In that range of inequality, it is always true that $\widetilde{\tau}_{\{h, m\}}<\widetilde{\tau}_{\{h, m, l\}}$. In that case, the club $\{h, m\}$ blocks the partition $P_{t}^{I}=(\{h, m, l\})$ because $\ln G_{j t}$ available to agents of type $h$, and $m$ in the partition $P_{t}^{I I}=(\{h, m\} ;\{l\})$ is higher compared to the level of public good they could benefit in the partition $P_{t}^{I}=(\{h, m, l\})$; that is,

$\frac{n\left(1-\tilde{\tau}_{\{h, m, l\}}\right)\left(h_{t}^{h}+h_{t}^{m}\right)+2 n\left(\tilde{\tau}_{\{h, m, l\}}-\tilde{\tau}_{\{h, m, l\}}^{2}\right) \bar{h}_{t}}{2 n+\beta}<\frac{n\left(1-\tilde{\tau}_{\{h, m\}}\right)\left(h_{t}^{h}+h_{t}^{m}\right)+2 n\left(\tilde{\tau}_{\{h, m\}}-\tilde{\tau}_{\{h, m\}}^{2}\right) \bar{h}_{t}}{2 n+\beta}$

Finally, notice that by definition, $\widetilde{\tau}_{\{h, m, l\}}=\frac{n\left(h_{t}^{h}+h_{t}^{m}\right)-(2 n+\beta) h_{t}^{l}}{\beta \bar{h}_{t}}$. Hence, in that range of inequality where $h_{t}^{h} \geq$ $\frac{(n+\beta)}{n} h_{t}^{m} \geq\left(\frac{n+\beta}{n}\right)^{2} h_{t}^{l}$, it is easily checked that $\tilde{\tau}_{\{h, m, l\}}>1$.

Proof of Proposition 2 Suppose that the ex ante pattern of income distribution is such that: (i) $0 \leq \tilde{\tau}_{\{h, m\}} \leq \tilde{\tau}_{\{m, l\}}$, 
(ii) $\tilde{\tau}_{\{h, m\}} \leq \tau_{\{l\}}^{*}$, and (iii) $V_{t}^{m}\left(\{h, m\} ; \tilde{\tau}_{\{h, m\}}\right)>V_{t}^{m}\left(\{m\} ; \tau_{\{m\}}^{*}\right)$, are satisfied simultaneously. First, (i) and (ii) imply that $\tilde{\tau}_{\{h, m\}}$ defeats in binary contest the candidates $\tau_{\{l\}}^{*}$ and $\widetilde{\tau}_{\{m, l\}}$. Second, consider the case where $\tilde{\tau}_{\{h, m\}}>\tau_{\{m, l\}}^{*}$, then (i) implies that the club $\{m, l\}$ is unfeasible with $\tau_{\{m, l\}}^{*}$. Provided that (ii) and (iii) are also satisfied, the preferences for both the low- and the middle-income classes are, $V_{t}^{l}\left(\{l\} ; \tilde{\tau}_{\{h, m\}}\right)>V_{t}^{l}\left(\{l\} ; \tau_{\{m, l\}}^{*}\right)$, respectively $V_{t}^{m}\left(\{h, m\} ; \tilde{\tau}_{\{h, m\}}\right)>V_{t}^{m}\left(\{m\} ; \tau_{\{m\}}^{*}\right)>V_{t}^{m}\left(\{m\} ; \tau_{\{m, l\}}^{*}\right)$. On the other hand, $\widetilde{\tau}_{\{h, m\}} \leq \tau_{\{m, l\}}^{*}$ yields the following preference ordering for the middle-income class: $V_{t}^{m}\left(\{h, m\} ; \tilde{\tau}_{\{h, m\}}\right) \geq V_{t}^{m}\left(\{h, m\} ; \tau_{\{m, l\}}^{*}\right)$. Therefore, (i), (ii), and (iii) also lead $\widetilde{\tau}_{\{h, m\}}$ to defeat $\tau_{\{m, l\}}^{*}$ in pairwise comparison whatever $\widetilde{\tau}_{\{h, m\}} \gtreqless \tau_{\{m, l\}}^{*}$. Finally, using Lemma 1 and considering the above result and (iii) allows us to infer that the middle-income class is best off in the club $\{h, m\}$ associated with $\tilde{\tau}_{\{h, m\}}$. There is no other issue than $\tilde{\tau}_{\{h, m\}}$ which is preferred by both the high- and the low-income groups. Therefore, in that range of ex ante inequality, the equilibrium partition is $P_{t}^{I I}=(\{h, m\} ;\{l\})$ associated with $\tilde{\tau}_{\{h, m\}} \geq 0$.

The following lemma allows us to prove Propositions 3 and 4.

Lemma 2 The middle-income class may be indifferent between clubs $\{m, l\}$ and $\{m\}$ associated with tax rate $\tau_{\{m, l\}}^{*}$, respectively $\tau_{\{m\}}^{*}$; that is, $V_{t}^{m}\left(\{m, l\}, \tau_{\{m, l\}}^{*}\right)=V_{t}^{m}\left(\{m\}, \tau_{\{m\}}^{*}\right)$, if and only if the ex ante pattern of inequality is such that: $\tau_{\{m\}}^{*}<\tilde{\tau}_{\{m, l\}}<\tau_{\{m, l\}}^{*}$.

Proof. By definition, notice that $V_{t}^{m}\left(\{m, l\} ; \tilde{\tau}_{\{m, l\}}\right)-V_{t}^{m}\left(\{m\} ; \tilde{\tau}_{\{m, l\}}\right)=0$. Then, for any tax rate $\tau>\widetilde{\tau}_{\{m, l\}}$, the middle-income class is better off in the club $\{m, l\}$ compared to the club $\{m\}$. On the one hand, if $\tilde{\tau}_{\{m, l\}} \leq \tau_{\{m\}}^{*}$, it is obvious that we have $V_{t}^{m}\left(\{m\}, \tau_{\{m\}}^{*}\right)<V_{t}^{m}\left(\{m, l\}, \tau_{\{m\}}^{*}\right)<V_{t}^{m}\left(\{m, l\}, \tau_{\{m, l\}}^{*}\right)$. On the other hand, when $\tilde{\tau}_{\{m, l\}} \geq \tau_{\{m, l\}}^{*}$, we also always have $V_{t}^{m}\left(\{m\}, \tau_{\{m\}}^{*}\right)>V_{t}^{m}\left(\{m\}, \tau_{\{m, l\}}^{*}\right)>V_{t}^{m}\left(\{m, l\}, \tau_{\{m, l\}}^{*}\right)$.

Proof of Proposition 3 Consider an ex ante pattern of income distribution such that $V_{t}^{m}\left(\{m\} ; \tau_{\{m\}}^{*}\right)>V_{t}^{m}\left(\{m, l\} ; \tau_{\{m, l\}}^{*}\right.$, Using Lemma 2, it is straightforward that $\tilde{\tau}_{\{m, l\}}>\tau_{\{m\}}^{*}$ and therefore $\tau_{\{m\}}^{*}$ defeats in binary contest $\tilde{\tau}_{\{m, l\}}$, $\tau_{\{m, l\}}^{*}$, and a fortiori $\tau_{\{l\}}^{*}$. Therefore, the only trade off faced by the middle-income class is between the candidates $\tau_{\{m\}}^{*}$ and $\widetilde{\tau}_{\{h, m\}}$. Two possibilities may arise. First, the pattern of inequality is such that $V_{t}^{m}\left(\{m\} ; \tau_{\{m\}}^{*}\right) \geq$ $V_{t}^{m}\left(\{h, m\} ; \tilde{\tau}_{\{h, m\}}\right)$ which implies that $\tilde{\tau}_{\{h, m\}}>\tau_{\{m\}}^{*}$, and whatever $V_{t}^{l}\left(\{l\} ; \tau_{\{m\}}^{*}\right) \gtreqless V_{t}^{l}\left(\{l\} ; \tilde{\tau}_{\{h, m\}}\right)$, the 
Condorcet winner is $\tau_{\{m\}}^{*}$. Second, the ex ante income distribution is such that $V_{t}^{m}\left(\{m\} ; \tau_{\{m\}}^{*}\right)<V_{t}^{m}\left(\{h, m\} ; \tilde{\tau}_{\{h, m\}}\right)$ but $V_{t}^{l}\left(\{l\} ; \tau_{\{m\}}^{*}\right)>V_{t}^{l}\left(\{l\} ; \widetilde{\tau}_{\{h, m\}}\right)$. This pattern of preferences may occur either when $\widetilde{\tau}_{\{h, m\}}<\tau_{\{m\}}^{*}$, or when $\tilde{\tau}_{\{h, m\}}>\tau_{\{m\}}^{*}$. Let us consider the case where $\tilde{\tau}_{\{h, m\}}<\tau_{\{m\}}^{*}$, and notice that the starting condition $V_{t}^{m}\left(\{m\} ; \tau_{\{m\}}^{*}\right)>V_{t}^{m}\left(\{m, l\} ; \tau_{\{m, l\}}^{*}\right)$ implies that $\tilde{\tau}_{\{m, l\}}>\tau_{\{m\}}^{*}$ which in turn implies that $\tilde{\tau}_{\{h, m\}}<$ $\tilde{\tau}_{\{m, l\}}$. Then, we are back to the situation described in Proposition 2. On the other hand, when $\tilde{\tau}_{\{h, m\}}>\tau_{\{m\}}^{*}$ and $V_{t}^{l}\left(\{l\} ; \tau_{\{m\}}^{*}\right)>V_{t}^{l}\left(\{l\} ; \widetilde{\tau}_{\{h, m\}}\right)$, both the low- and the high-income classes prefer $\tau_{\{m\}}^{*}$ compared to $\tilde{\tau}_{\{h, m\}}$. Finally, notice that in these cases, $\widetilde{\tau}_{\{h, m\}}$ and $\widetilde{\tau}_{\{m, l\}}$ are both strictly positive. Thus, using Lemma 1, we can ignore the candidate $\tilde{\tau}_{\{h, m, l\}}$. Hence, in that range of inequality, the Condorcet winner is $\tau_{\{m\}}^{*} \geq 0$ which yields the equilibrium partition $P_{t}^{I I I}=(\{h\} ;\{m\} ;\{l\})$.

The proof of Proposition 4 requires another lemma.

Lemma 3 The middle-income class may be indifferent between clubs $\{m, l\}$ and $\{m\}$ associated with tax rate $\tilde{\tau}_{\{h, m\}}$, respectively $\tau_{\{m\}}^{*}$; that is, $V_{t}^{m}\left(\{m, l\}, \widetilde{\tau}_{\{h, m\}}\right)=V_{t}^{m}\left(\{m\}, \tau_{\{m\}}^{*}\right)$, when the ex ante pattern of inequality is such that $\tau_{\{m\}}^{*} \leq \tilde{\tau}_{\{m, l\}} \leq \tilde{\tau}_{\{h, m\}} \leq \tau_{\{m, l\}}^{*}$

Proof. Recall that by definition, we always have $V_{t}^{m}\left(\{m, l\} ; \tilde{\tau}_{\{m, l\}}\right)-V_{t}^{m}\left(\{m\} ; \tilde{\tau}_{\{m, l\}}\right)=0$. Hence, when $\tau_{\{m\}}^{*} \leq$ $\tilde{\tau}_{\{m, l\}}$ we also have $V_{t}^{m}\left(\{m\}, \tau_{\{m\}}^{*}\right) \geq V_{t}^{m}\left(\{m, l\} ; \tilde{\tau}_{\{m, l\}}\right)$. Using Lemma 2 , it is easily checked that in this range of inequality, there may exist $\widetilde{\tau}_{\{m, l\}}$ such that $V_{t}^{m}\left(\{m, l\}, \tau_{\{m, l\}}^{*}\right) \geq V_{t}^{m}\left(\{m\}, \tau_{\{m\}}^{*}\right)$. In that case, because $\tilde{\tau}_{\{h, m\}} \leq$ $\tau_{\{m, l\}}^{*}$, there also exists $\tilde{\tau}_{\{h, m\}}$ such that $V_{t}^{m}\left(\{m, l\}, \tilde{\tau}_{\{h, m\}}\right)=V_{t}^{m}\left(\{m\}, \tau_{\{m\}}^{*}\right)$

Proof of Proposition 4 When the ex ante pattern of income distribution is such that: (i) $\tilde{\tau}_{\{h, m\}} \geq \tau_{\{m, l\}}^{*}$, (ii) $V_{t}^{m}\left(\{m\} ; \tau_{\{m\}}^{*}\right) \leq V_{t}^{m}\left(\{m, l\} ; \tau_{\{m, l\}}^{*}\right)$ and (iii) $V_{t}^{i}\left(\{m, l\} ; \tau_{\{m, l\}}^{*}\right)>V_{t}^{i}\left(\{h, m, l\} ; \widetilde{\tau}_{\{h, m, l\}}\right)$ for $i=m, l$ are satisfied simultaneously, notice first that $\tau_{\{m, l\}}^{*}$ is strictly preferred by a majority to 0 and $\tau_{\{m\}}^{*}$. From (ii) and using Lemma 2, we know that $0 \leq \tilde{\tau}_{\{m, l\}}<\tau_{\{m, l\}}^{*}$ which leads the club $\{m, l\}$ to be feasible with tax rate $\tau_{\{m, l\}}^{*}$ which, by definition, maximizes the level of public good in that club. Hence, $\tau_{\{m, l\}}^{*}$ defeats in binary contest $\widetilde{\tau}_{\{m, l\}}$ and $\tau_{\{l\}}^{*}$. Second, provided that (i) is satisfied, $\tau_{\{m, l\}}^{*}$ is always strictly preferred to $\widetilde{\tau}_{\{h, m\}}$ by the low- and the high-income classes although the middle-income class may prefer to form a club $\{h, m\}$ associated with $\tilde{\tau}_{\{h, m\}}$. Finally, given (i) and (iii), $\tau_{\{m, l\}}^{*}$ is unanimously preferred to $\widetilde{\tau}_{\{h, m, l\}}$. Thus, in that range of ex ante income distribution, the equilibrium 
partition is $P_{t}^{I V}=(\{h\} ;\{m, l\})$ associated with $\tau_{\{m, l\}}^{*}$.

This equilibrium partition also occurs when (i) $\tau_{\{m, l\}}^{*} \geq \widetilde{\tau}_{\{h, m\}}>\widetilde{\tau}_{\{m, l\}} \geq 0$, (ii) $V_{t}^{m}\left(\{m, l\}, \widetilde{\tau}_{\{h, m\}}\right)>$ $V_{t}^{m}\left(\{m\}, \tau_{\{m\}}^{*}\right)$, and (iii) $V_{t}^{i}\left(\{m, l\} ; \tilde{\tau}_{\{h, m\}}\right)>V_{t}^{i}\left(\{h, m, l\} ; \tilde{\tau}_{\{h, m, l\}}\right)$ for $i=m, l$ are satisfied simultaneously. Let us first consider the case $0<\widetilde{\tau}_{\{h, m\}} \leq \tau_{\{m\}}^{*}$, then it is clear that $\tilde{\tau}_{\{h, m\}}$ defeats in binary contest $\tau_{\{m\}}^{*}, \tau_{\{m, l\}}^{*}$, $\tau_{\{l\}}^{*}$, and 0 . The level of redistribution therefore lies between $\tilde{\tau}_{\{m, l\}} \geq 0$, and $\widetilde{\tau}_{\{h, m\}}$. Notice that the level of public good available in club $\{m, l\}$ increases in that range with the level of redistribution. $\tau=\frac{n h^{h}-(n+\beta) h^{m}}{\beta \bar{h}}>\widetilde{\tau}_{\{m, l\}}$ is the tax rate which provides the highest level of public good in club $\{m, l\}$ and which prevents the middle-income class to join the high-income class in a club $\{h, m\}$. Hence, $\tau=\frac{n h^{h}-(n+\beta) h^{m}}{\beta \bar{h}}$ is strictly preferred by both the low- and the high-income classes to $\widetilde{\tau}_{\{h, m\}}$. Second, when $\widetilde{\tau}_{\{h, m\}}>\tau_{\{m\}}^{*}$, provided that (ii) is satisfied and using Lemma 3, the same reasoning as above leads $\frac{n h^{h}-(n+\beta) h^{m}}{\beta \bar{h}}$ to be strictly preferred to $\widetilde{\tau}_{\{h, m\}}$. Finally, as long as (iii) is satisfied $\frac{n h^{h}-(n+\beta) h^{m}}{\beta \bar{h}}$ is a Condorcet winner in that range of inequality and the equilibrium partition is $P_{t}^{I V}=(\{h\} ;\{m, l\})$.

Finally, the following lemma provides information used to prove Proposition 5.

Lemma 4 Let the middle-income class be indifferent between $\tilde{\tau}_{\{h, m\}}$ and $\tau_{\{m\}}^{*}$ associated with club $\{h, m\}$, respectively $\{m\}$; that is, $V_{t}^{m}\left(\{h, m\} ; \widetilde{\tau}_{\{h, m\}}\right)=V_{t}^{m}\left(\{m\} ; \tau_{\{m\}}^{*}\right)$ and suppose that this indifference requires that $\widetilde{\tau}_{\{h, m\}}>$ $\tau_{\{l\}}^{*}$ for $\tau_{\{m\}}^{*}>0$. Then, as long as $V_{t}^{m}\left(\{h, m\} ; \tilde{\tau}_{\{h, m\}}\right)>V_{t}^{m}\left(\{m\} ; \tau_{\{m\}}^{*}\right)$, we also have $V_{t}^{m}\left(\{h, m\} ; \tau_{\{l\}}^{*}\right)>$ $V_{t}^{m}\left(\{m\} ; \tau_{\{m\}}^{*}\right)$.

\section{Proof. See Appendix B}

Proof of Proposition 5 All remaining ex ante patterns of income distribution yield Condorcet cycles. Keep in mind that whenever, $\widetilde{\tau}_{\{h, m\}}$ and $\widetilde{\tau}_{\{m, l\}}$ are both positive we can ignore $\widetilde{\tau}_{\{h, m, l\}}$ because all income classes strictly prefer any other candidate.

- In Proposition 4, we just showed that $(\{h\} ;\{m, l\})$ emerges as an equilibrium partition when (i) $\tau_{\{m, l\}}^{*} \geq$ $\tilde{\tau}_{\{h, m\}}>\widetilde{\tau}_{\{m, l\}} \geq 0$, (ii) $V_{t}^{m}\left(\{m, l\}, \tilde{\tau}_{\{h, m\}}\right)>V_{t}^{m}\left(\{m\}, \tau_{\{m\}}^{*}\right)$, and (iii) $V_{t}^{i}\left(\{m, l\} ; \tilde{\tau}_{\{h, m\}}\right)>V_{t}^{i}\left(\{h, m, l\} ; \tilde{\tau}_{\{h, m, l\}}\right)$ for $i=m, l$ are satisfied simultaneously. As soon as (iii) is not satisfied $\tilde{\tau}_{\{h, m, l\}}$ becomes a key competitor compared to $\frac{n h^{h}-(n+\beta) h^{m}}{\beta \bar{h}}$. Indeed, in that case, we have

$$
V^{h}(\{h\} ; \tau)>V^{h}\left(\{h, m\} ; \tilde{\tau}_{\{h, m\}}\right)>V^{h}\left(\{h, m, l\} ; \tilde{\tau}_{\{h, m, l\}}\right)
$$




$$
\begin{gathered}
V^{m}\left(\{h, m\} ; \tilde{\tau}_{\{h, m\}}\right)>V^{m}\left(\{h, m, l\} ; \tilde{\tau}_{\{h, m, l\}}\right)>V^{m}(\{m, l\} ; \tau) \\
V^{l}\left(\{h, m, l\} ; \tilde{\tau}_{\{h, m, l\}}\right)>V^{l}(\{m, l\} ; \tau)>V^{l}\left(\{l\} ; \tilde{\tau}_{\{h, m\}}\right)
\end{gathered}
$$

with $\tau=\frac{n h^{h}-(n+\beta) h^{m}}{\beta \bar{h}}$.

Notice that the same is true when (i) $\tilde{\tau}_{\{h, m\}} \geq \tau_{\{m, l\}}^{*}$, (ii) $V_{t}^{m}\left(\{m\} ; \tau_{\{m\}}^{*}\right) \leq V_{t}^{m}\left(\{m, l\} ; \tau_{\{m, l\}}^{*}\right)$, but (iii) $V_{t}^{i}\left(\{m, l\} ; \tau_{\{m, l\}}^{*}\right) \leq V_{t}^{i}\left(\{h, m, l\} ; \tilde{\tau}_{\{h, m, l\}}\right)$ for $i=m, l$. In that case, $\tilde{\tau}_{\{h, m, l\}}$ becomes a key competitor compared to $\tau_{\{m, l\}}^{*}$ which is not anymore a Condorcet winner.

- A second type of cycle occurs when (i) $\tau_{\{m\}}^{*} \leq \tilde{\tau}_{\{m, l\}}<\tilde{\tau}_{\{h, m\}}<\tau_{\{m, l\}}^{*}$, and (ii) $V_{t}^{m}\left(\{m, l\}, \tilde{\tau}_{\{h, m\}}\right) \leq$ $V_{t}^{m}\left(\{m\}, \tau_{\{m\}}^{*}\right)$ are satisfied simultaneously. Notice, that this range of inequality is very similar to the above case described in Proposition 4 except that (ii) implies now that $\tau_{\{m\}}^{*}$ becomes a serious competitor in pairwise comparison to $\frac{n h^{h}-(n+\beta) h^{m}}{\beta \bar{h}}$. Indeed, in that case we have the following ordering of relevant preferences which yields a Condorcet cycle:

$$
\begin{gathered}
V^{h}\left(\{h\} ; \tau_{\{m\}}^{*}\right)>V^{h}(\{h\} ; \tau)>V^{h}\left(\{h, m\} ; \tilde{\tau}_{\{h, m\}}\right) \\
V^{m}\left(\{h, m\} ; \tilde{\tau}_{\{h, m\}}\right)>V^{m}\left(\{m\} ; \tau_{\{m\}}^{*}\right)>V^{m}(\{m, l\} ; \tau) \\
V^{l}(\{m, l\} ; \tau)>V^{l}\left(\{l\} ; \tilde{\tau}_{\{h, m\}}\right)>V^{l}\left(\{l\} ; \tau_{\{m\}}^{*}\right)
\end{gathered}
$$

with $\tau=\frac{n h^{h}-(n+\beta) h^{m}}{\beta \bar{h}}$.

- A third type of cycle occurs when (i) $\tau_{\{m, l\}}^{*} \leq \tilde{\tau}_{\{h, m\}} \leq \tau_{\{l\}}^{*}$, (ii) $\tilde{\tau}_{\{h, m\}}>\tilde{\tau}_{\{m, l\}}$, (iii) $V_{t}^{m}\left(\{m\} ; \tau_{\{m\}}^{*}\right)>$ $V_{t}^{m}\left(\{m, l\} ; \tau_{\{m, l\}}^{*}\right)$, and (iv) $V_{t}^{m}\left(\{h, m\} ; \widetilde{\tau}_{\{h, m\}}\right)>V_{t}^{m}\left(\{m\} ; \tau_{\{m\}}^{*}\right)$ are satisfied simultaneously. In that range of inequality, the preferences of the different income groups are either as displayed in Figure 3 in the text where the most preferred tax rate of the low-income class is $\tau_{\{m, l\}}^{*}$, i.e., $\tilde{\tau}_{\{m, l\}} \leq \tau_{\{m, l\}}^{*}$, or such that the most preferred tax rate of the low-income class is $\widetilde{\tau}_{\{m, l\}}$, i.e., $\widetilde{\tau}_{\{m, l\}}>\tau_{\{m, l\}}^{*}$, everything else being equal. In that case, it can easily be checked that there is no Condorcet winner. For instance, using Lemma 4, when $\tilde{\tau}_{\{m, l\}} \leq \tau_{\{m, l\}}^{*}$, the preference ordering of the different relevant candidates for each income class is as follows,

$$
\begin{gathered}
V^{h}\left(\{h\} ; \tau_{\{m\}}^{*}\right)>V^{h}\left(\{h\} ; \tau_{\{m, l\}}^{*}\right)>V^{h}\left(\{h, m\} ; \tilde{\tau}_{\{h, m\}}\right)>V^{h}\left(\{h, m\} ; \tau_{\{l\}}^{*}\right) \\
V^{m}\left(\{h, m\} ; \widetilde{\tau}_{\{h, m\}}\right)>V^{m}\left(\{h, m\} ; \tau_{\{l\}}^{*}\right)>V^{m}\left(\{m\} ; \tau_{\{m\}}^{*}\right)>V^{m}\left(\{m, l\} ; \tau_{\{m, l\}}^{*}\right)
\end{gathered}
$$




$$
V^{l}\left(\{m, l\} ; \tau_{\{m, l\}}^{*}\right)>V^{l}\left(\{l\} ; \tau_{\{l\}}^{*}\right)>V^{l}\left(\{l\} ; \tilde{\tau}_{\{h, m\}}\right)>V^{l}\left(\{l\} ; \tau_{\{m\}}^{*}\right)
$$

On the other hand, when $\widetilde{\tau}_{\{m, l\}}>\tau_{\{m, l\}}^{*}$ everything else being equal, the preference ordering is the same as above but the club $\{m, l\}$ becomes now unfeasible, and the same type of Condorcet paradox occurs where $V^{i}\left(S_{j t} ; \tau_{\{m, l\}}^{*}\right)$ must be replaced by $V^{i}\left(S_{j t} ; \tilde{\tau}_{\{m, l\}}\right)$.

Let us now consider an ex ante pattern of income distribution defined as above where (ii), (iii), and (iv) are satisfied, but where $\tilde{\tau}_{\{h, m\}}>\tau_{\{l\}}^{*}$. Then, the candidate $\tau_{\{l\}}^{*}$ which now yields the partition $(\{h\} ;\{m, l\})$ is defeated for each income class by $\tau_{\{m, l\}}^{*}$, when $\tilde{\tau}_{\{m, l\}} \leq \tau_{\{m, l\}}^{*}$ and by $\tilde{\tau}_{\{m, l\}}$ when $\tau_{\{l\}}^{*}>\widetilde{\tau}_{\{m, l\}}>\tau_{\{m, l\}}^{*}$. In both these cases, there are Condorcet paradoxes between $\tilde{\tau}_{\{h, m\}}, \tau_{\{m\}}^{*}$, and either $\tau_{\{m, l\}}^{*}$ or $\tilde{\tau}_{\{m, l\}}$ as long as (v) $V^{l}\left(\{l\} ; \tilde{\tau}_{\{h, m\}}\right)>$ $V^{l}\left(\{l\} ; \tau_{\{m\}}^{*}\right)$ is satisfied. Similarly, a Condorcet cycle arises when $\tilde{\tau}_{\{m, l\}} \geq \tau_{\{l\}}^{*}$ as long as $V^{l}\left(\{l\} ; \tilde{\tau}_{\{h, m\}}\right)>$ $V^{l}\left(\{l\} ; \tau_{\{m\}}^{*}\right)$. More specifically, we have

$$
\begin{gathered}
V^{h}\left(\{h\} ; \tau_{\{m\}}^{*}\right)>V^{h}(\{h\} ; \tau)>V^{h}\left(\{h, m\} ; \tilde{\tau}_{\{h, m\}}\right) \\
V^{m}\left(\{h, m\} ; \tilde{\tau}_{\{h, m\}}\right)>V^{m}\left(\{m\} ; \tau_{\{m\}}^{*}\right)>V^{m}(\{m, l\} ; \tau) \\
V^{l}(\{m, l\} ; \tau)>V^{l}\left(\{l\} ; \tilde{\tau}_{\{h, m\}}\right)>V^{l}\left(\{l\} ; \tau_{\{m\}}^{*}\right)
\end{gathered}
$$

with $\tau=\tau_{\{m, l\}}^{*}$, when $\tilde{\tau}_{\{m, l\}} \leq \tau_{\{m, l\}}^{*}, \tau=\tilde{\tau}_{\{m, l\}}$ when $\tau_{\{l\}}^{*}>\tilde{\tau}_{\{m, l\}}>\tau_{\{m, l\}}^{*}$, and $\tau=\tilde{\tau}_{\{m, l\}}$, or $\tau=\tau_{\{l\}}^{*}$ when $\tilde{\tau}_{\{m, l\}}>\tau_{\{l\}}^{*}$ depending on whether $V^{l}\left(\{m, l\} ; \tilde{\tau}_{\{m, l\}}\right) \gtreqless V^{l}\left(\{l\} ; \tau_{\{l\}}^{*}\right)$.

Finally, in that range of inequality where (iii), (iv), and (v) are still satisfied simultaneously, but where $\widetilde{\tau}_{\{h, m\}} \leq$ $\tilde{\tau}_{\{m, l\}}$ and $\tilde{\tau}_{\{h, m\}}>\tau_{\{l\}}^{*}$, a Condorcet cycle also occurs between the candidates $\tau_{\{m\}}^{*}, \tilde{\tau}_{\{h, m\}} \tau_{\{l\}}^{*}$. Notice that, everything else being equal, whenever $V^{l}\left(\{l\} ; \tilde{\tau}_{\{h, m\}}\right) \leq V^{l}\left(\{l\} ; \tau_{\{m\}}^{*}\right)$, we are back to Proposition 3 .

The discussion is now complete as we considered all the possible rankings of the potential candidates as well as their underlying preference orderings for the three different income classes whatever the ex ante initial pattern of income distribution is.

\section{Appendix B. Construction of Figure 4}

This appendix provides some information about the construction of Figure 4. From now on, we denote $x_{t}=h_{t}^{h} / h_{t}^{m}$, 
$y_{t}=h_{t}^{m} / h_{t}^{l}, \eta=\frac{n}{\beta}$, and consider that $\eta>6$.

1. First, notice that the zero-tax rate locus $\tau_{\{m\}}^{*}=0$ is such that $y_{t}=\frac{1}{2-x_{t}}$. This locus is defined for $x_{t} \in[1,2[$, upward sloping and convex. The display of the zero-tax rates loci $\widetilde{\tau}_{\{h, m, l\}}=0, \widetilde{\tau}_{\{h, m\}}=0, \widetilde{\tau}_{\{m, l\}}=0$ is also straightforward.

Second, by definition, the locus $\widetilde{\tau}_{\{h, m\}}=\widetilde{\tau}_{\{m, l\}}>0$ is given by all pairs $\left(x_{t}, y_{t}\right)$ that satisfy the following equality:

$$
y_{t}=\frac{\eta+1}{2 \eta+1-\eta x_{t}}
$$

It is defined in $\left[1+\eta^{-1}, 2+\eta^{-1}[\right.$, upward sloping and convex.

Finally, given the definition of the different tax rates, a similar reasoning allows us to depict the loci $\widetilde{\tau}_{\{h, m\}}=\tau_{\{l\}}^{*}$, and $\widetilde{\tau}_{\{h, m\}}=\tau_{\{m, l\}}^{*}$ as displayed in Figure 4 and to show that the locus $\tau_{\{m\}}^{*}=0$ is always located above the locus $\tilde{\tau}_{\{h, m\}}=\tilde{\tau}_{\{m, l\}}$.

2. First, we are now interested in the loci comparing indifferent welfare levels between different community's structures for our three income classes. We provide information about the Locus $A A$ in Figure 4 which characterizes the initial pattern of income inequality such that $V_{t}^{m}\left(\{m\} ; \tau_{\{m\}}^{*}\right)=V_{t}^{m}\left(\{h, m\} ; \tilde{\tau}_{\{h, m\}}\right)$.

Notice that this locus belongs to the region of the plane where $\tilde{\tau}_{\{h, m\}}>0$, i.e. $x_{t}>1+\eta^{-1}$ and $y_{t} \geq 1$.

Given the definitions of $\tau_{\{m\}}^{*}$, and $\widetilde{\tau}_{\{h, m\}}$, this locus is defined as

$$
\frac{n\left(1-\tau_{\{m\}}^{*}\right) h_{t}^{m}+n\left(\tau_{\{m\}}^{*}-\left(\tau_{\{m\}}^{*}\right)^{2}\right) \bar{h}_{t}}{n+\beta}=\frac{n\left(1-\widetilde{\tau}_{\{h, m\}}\right) h_{t}^{h}+n\left(\widetilde{\tau}_{\{h, m\}}-\left(\widetilde{\tau}_{\{h, m\}}\right)^{2}\right) \bar{h}_{t}}{n+\beta}
$$

When $\tau_{\{m\}}^{*}>0$, replacing $\tau_{\{m\}}^{*}$ and $\widetilde{\tau}_{\{h, m\}}$ by their expressions yields an implicit function $f\left(x_{t}, y_{t}\right)=0$ which is downward sloping in the plane $\left(x_{t}, y_{t}\right)$. Indeed, notice that $f\left(x_{t}, y_{t}\right)=0$ has two real roots $x_{t}^{\prime}$ and $x_{t}^{\prime \prime}$

$$
x_{t}^{\prime}=A+B y_{t}^{-1} \text { or } x_{t}^{\prime \prime}=C+D y_{t}^{-1}
$$

As the solution $A+B y_{t}^{-1}$ is defined in our plane where $\widetilde{\tau}_{\{h, m\}} \leq 0$, we only take into consideration the locus

$$
x_{t}=C+D y_{t}^{-1}
$$

where $C=-\left(11-36 \eta^{2}-24 \eta\right)^{-1}\left(36 \eta^{2}+54 \eta+30 \sqrt{(1+\eta)}+14\right)$, 
and $D=-\left(11-36 \eta^{2}-24 \eta\right)^{-1}(6 \eta+6 \sqrt{(1+\eta)}+5)$.

We can easily conclude that it is monotonically decreasing and convex. On the other hand, considering initial patterns of income distribution such that $\tau_{m}^{*}=0$, i.e. $h_{t}^{m} \geq \bar{h}_{t}$, the locus $V_{t}^{m}\left(\{m\} ; \tau_{\{m\}}^{*}\right)=V_{t}^{m}\left(\{h, m\} ; \tilde{\tau}_{\{h, m\}}\right)$ becomes

$$
\frac{n h_{t}^{m}}{n+\beta}=\frac{n\left(1-\tilde{\tau}_{\{h, m\}}\right) h_{t}^{h}+n\left(\widetilde{\tau}_{\{h, m\}}-\left(\widetilde{\tau}_{\{h, m\}}\right)^{2}\right) \bar{h}_{t}}{n+\beta}
$$

Replacing $\widetilde{\tau}_{\{h, m\}}$ by its definition, similar straightforward algebra allows us to conclude that this locus is monotonically decreasing and convex in our plane.

Second, recall that we assumed in Lemma 4 that the locus $V_{t}^{m}\left(\{m\} ; \tau_{\{m\}}^{*}\right)=V_{t}^{m}\left(\{h, m\} ; \tilde{\tau}_{\{h, m\}}\right)$ is always located to the right of the locus $\widetilde{\tau}_{\{h, m\}}=\tau_{\{l\}}^{*}$ when $\tau_{\{m\}}^{*}>0$. We now prove this result. On the one hand, both the loci $\tau_{\{m\}}^{*}=(1 / 2)\left(1-h_{t}^{m} / \bar{h}_{t}\right)=0$ and $V_{t}^{m}\left(\{m\} ; \tau_{\{m\}}^{*}\right)=V_{t}^{m}\left(\{h, m\} ; \widetilde{\tau}_{\{h, m\}}\right)$ intersect where

$$
x_{t}=\left(6 \eta^{2}+11 \eta+7 \sqrt{(1+\eta)}+4\right)\left(6 \eta^{2}+5 \eta+\sqrt{(1+\eta)}-1\right)^{-1}
$$

On the other hand, the locus $\tau_{\{m\}}^{*}=(1 / 2)\left(1-h_{t}^{m} / \bar{h}_{t}\right)=0$ and the locus $\widetilde{\tau}_{\{h, m\}}=\tau_{\{l\}}^{*}$ intersect where

$$
x_{t}=\left(\eta+\frac{1}{2}\right)\left(\eta-\frac{1}{2}\right)^{-1}
$$

which is smaller than the prior intersection for any $\eta \geq 1$. Because $\widetilde{\tau}_{\{h, m\}}=\tau_{\{l\}}^{*}$, respectively $V_{t}^{m}\left(\{m\} ; \tau_{\{m\}}^{*}\right)=$ $V_{t}^{m}\left(\{h, m\} ; \tilde{\tau}_{\{h, m\}}\right)$, is increasing, respectively decreasing, the locus $V_{t}^{m}\left(\{m\} ; \tau_{\{m\}}^{*}\right)=V_{t}^{m}\left(\{h, m\} ; \tilde{\tau}_{\{h, m\}}\right)$ is always located to the right of the locus $\tilde{\tau}_{\{h, m\}}=\tau_{\{l\}}^{*}$ in our plane as long as the initial pattern of income inequality is such that $\tau_{\{m\}}^{*}>0$.

3. Finally, some tedious but straightforward algebra allows us to depict in Figure 4 the other loci as defined in Footnote 6 in the text. Further information about the construction of Figure 4 is available upon request.

\section{Appendix C. Dynamics of Inequality}

\section{Evolving income distribution}


(i) When $\left(x_{t}, y_{t}\right) \in$ Region $I$, we have

$$
\left\{\begin{array}{l}
x_{t+1}=\left(x_{t}\right)^{1-\alpha-\beta} \\
y_{t+1}=\left(y_{t}\right)^{1-\alpha-\beta}
\end{array}\right.
$$

(ii) When $\left(x_{t}, y_{t}\right) \in$ Region $I I$, we have the following equations

$$
\left\{\begin{array}{c}
x_{t+1}=\left(x_{t}\right)^{1-\alpha-\beta} \\
y_{t+1}=\left(y_{t}\right)^{1-\alpha-\beta}\left(\frac{x_{t}+\tilde{\tau}_{\{h, m\}}\left(x_{t}+y_{t}^{-1}+1\right) / 3}{y_{t}^{-1}+\tilde{\tau}_{\{h, m\}}\left(x_{t}+y_{t}^{-1}+1\right) / 3}\right)^{\beta}
\end{array}\right.
$$

(iii) When $\left(x_{t}, y_{t}\right) \in$ Region $I I I$, the dynamics of inequality are described by the following dynamical system

$$
\left\{\begin{array}{c}
x_{t+1}=\left(x_{t}\right)^{1-\alpha-\beta}\left(\frac{x_{t}+\tau_{\{m\}}^{*}\left(x_{t}+y_{t}^{-1}+1\right) / 3}{1+\tau_{\{m\}}^{*}\left(x_{t}+y_{t}^{-1}+1\right) / 3}\right)^{\beta} \\
y_{t+1}=\left(y_{t}\right)^{1-\alpha-\beta}\left(\frac{1+\tau_{\{m\}}^{*}\left(x_{t}+y_{t}^{-1}+1\right) / 3}{\left(1 / y_{t}\right)+\tau_{\{m\}}^{*}\left(x_{t}+y_{t}^{-1}+1\right) / 3}\right)^{\beta}
\end{array}\right.
$$

(iv) Finally, when $\left(x_{t}, y_{t}\right) \in$ Region $I V$, the dynamical system is

$$
\left\{\begin{array}{c}
x_{t+1}=\left(x_{t}\right)^{1-\alpha-\beta}\left(\frac{x_{t}+\tau\left(x_{t}+y_{t}^{-1}+1\right) / 3}{\eta+1}\right)^{\beta}\left(\frac{2 \eta+1}{1+1 / y_{t}+2 \tau\left(x_{t}+y_{t}^{-1}+1\right) / 3}\right)^{\beta} \\
y_{t+1}=\left(y_{t}\right)^{1-\alpha-\beta}
\end{array}\right.
$$

with $\tau=\tau_{\{m, l\}}^{*}$ if $\left(n h_{t}^{h}-(n+\beta) h_{t}^{m}\right) / \beta \bar{h}_{t}<\tau_{\{m, l\}}^{*}$ and $\tau=\left(n h_{t}^{h}-(n+\beta) h_{t}^{m}\right) / \beta \bar{h}_{t}$ otherwise.

As a consequence, the global dynamics that describe the evolution of the income distribution are such that,

Lemma 5 The sequence $\left\{x_{t}\right\}_{t=0}^{\infty}$ is monotonically decreasing in the plane $\left(x_{t}, y_{t}\right)$.

Proof. Recall that $\alpha+\beta<1$, and having in mind that $\frac{x_{t}+A}{1+A} \leq x_{t}$ for $A \geq 0$, it is then straightforward that $x_{t+1}<x_{t}$ everywhere in the plane $\left(x_{t}, y_{t}\right)$.

The sequence $\left\{y_{t}\right\}_{t=0}^{\infty}$ is slightly more intricate.

Lemma 6 The global dynamics of $y_{t}$ are given by

(i) if $\left(x_{t}, y_{t}\right) \in$ Region $I, I I I$, and $I V$, then $\frac{y_{t+1}}{y_{t}}<1$.

(ii) if $\left(x_{t}, y_{t}\right) \in$ Region $I I$, then

$$
\frac{y_{t+1}}{y_{t}}\left\{\begin{aligned}
<1 & \text { if }\left(x_{t}, y_{t}\right)>\left(x_{t}, y\left(x_{t}\right)\right) \\
=1 & \text { if }\left(x_{t}, y_{t}\right)=\left(x_{t}, y\left(x_{t}\right)\right) \\
>1 & \text { if }\left(x_{t}, y_{t}\right)<\left(x_{t}, y\left(x_{t}\right)\right)
\end{aligned}\right.
$$

where $y\left(x_{t}\right)$ is a single-valued function such that $\left(x_{t}, y\left(x_{t}\right)\right) \in\left\{\left(x_{t}, y_{t}\right) \mid \frac{y_{t+1}}{y_{t}}=1\right\}$.

Proof. (i) is straightforward. (ii) Let us focus on the locus $y\left(x_{t}\right)$ such that $\frac{y_{t+1}}{y_{t}}=1$ in Region $I I$. 
When $\tilde{\tau}_{\{h, m\}}>0$, it is such that

$$
y_{t}^{\frac{\alpha}{\beta}+1}=\frac{(\eta+1)(x-1)}{\eta x_{t}+y_{t}^{-1}-(\eta+1)}
$$

Using the implicit function theorem, it is easily shown that this locus is monotonically decreasing and convex for $x_{t} \in\left[1+\eta^{-1}, \infty\left[\right.\right.$. It corresponds to the locus $Y^{\prime} Y^{\prime}$ in Figure 6 in the text.

When $\widetilde{\tau}_{\{h, m\}}=0$, we have the following expression

$$
y_{t}^{\frac{\alpha}{\beta}}=\frac{(\eta+1)\left(x_{t}+1\right)}{2 \eta+1}
$$

and the corresponding locus denoted $Y Y$ in Figure 6 is monotonically increasing and convex for $\beta>\alpha$

Proof of Proposition 6 First, using the definition of $\tilde{\tau}_{\{h, m\}}$, the dynamics in Region $I I$ are the following

$$
\left\{\begin{array}{c}
x_{t+1}=\left(x_{t}\right)^{1-\alpha-\beta} \\
y_{t+1}=\left(y_{t}\right)^{1-\alpha}\left(\frac{\eta+1}{2 \eta+1}\right)^{\beta}\left(x_{t}+1\right)^{\beta}
\end{array}\right.
$$

Log-linearizing in the neighborhood of the steady state, $x_{\infty}=1$ et $y_{\infty}=\left(\frac{2(n+\beta)}{2 n+\beta}\right)^{\frac{\beta}{\alpha}}$, we obtain two real eigenvalues $\lambda_{1}=(1-\alpha-\beta)$ and $\lambda_{2}=(1-\alpha)$, both smaller than 1. It is then obvious that these dynamics are locally stable within Region $I I$. Notice that if and only if $\frac{\beta}{\alpha} \geq \Pi$, then the pair $\left(1,\left(\frac{2(n+\beta)}{2 n+\beta}\right)^{\frac{\beta}{\alpha}}\right)$ belongs to Region $I I$ and the global dynamics exhibit multiple steady states.

Second, it follows directly from the dynamics in Region $I$ defined above that the integrated steady state is stable.

Proof of Proposition 7 Let us denote $\gamma^{*}$ the growth rate at the integrated equilibrium which is equal to

$$
\gamma^{*}=\kappa\left(\frac{3 n \beta}{3 n+\beta}\right)^{\beta}
$$

While $\lambda^{*}$, the growth rate at the segregated equilibrium can be expressed by

$$
\lambda^{*}=\kappa\left[\left(\frac{2 n \beta}{2 n+\beta}\right)^{\beta} f+\left(\frac{n \beta}{n+\beta}\right)^{\beta} g\right]
$$

with $f=\frac{2}{3}\left(\frac{3 \bar{h}_{S_{j t}}}{2 \bar{h}_{S_{j t}}+h_{t}^{l}}\right)^{1-\alpha}, g=\frac{1}{3}\left(\frac{3 h_{t}^{l}}{2 \bar{h}_{S_{j t}}+h_{t}^{l}}\right)^{1-\alpha}$, and where $S_{j t}=\{h, m\}$.

Due to Jensen's inequality, we have $f+g=\frac{E\left(h_{t}^{1-\alpha}\right)}{\left[E\left(h_{t}\right)\right]^{1-\alpha}}<1$. We can easily conclude that $\gamma^{*}>\lambda^{*}$.

Proof of Proposition 8 Let us define $d_{t}$ the distance between a pair $\left(x_{t}, y_{t}\right)$ evolving in Region $I I$ where $x_{t} \leq$ 
$1+\eta^{-1}$ and the frontier between Regions $I$ and $I I\left(\widetilde{\tau}_{\{h, m, l\}}=0\right)$. It is given by

$$
d_{t}=y_{t}\left(\frac{2+\eta^{-1}}{1+x_{t}}\right)^{-1}
$$

We can rewrite the dynamical system in Region $I I$ where $x_{t} \leq 1+\eta^{-1}$ in the space $\left(x_{t}, d_{t}\right)$

$$
\left\{\begin{array}{c}
x_{t+1}=\left(x_{t}\right)^{1-\alpha-\beta} \\
d_{t+1}=\left(d_{t}\right)^{1-\alpha}\left(\frac{\eta+1}{2 \eta+1}\right)^{\beta}\left(\frac{1+\left(x_{t}\right)^{1-\alpha-\beta}}{\left(1+x_{t}\right)^{1-\alpha-\beta}}\right)\left(2+\eta^{-1}\right)^{-\alpha}
\end{array}\right.
$$

Notice that $d_{t+1}$ is increasing with $d_{t}$ and decreasing with $x_{t}$. Thus, $d_{t+1}$ is minimum for $d_{t}=1$ and $x_{t}=1+\eta^{-1}$. Then,

$$
\min \left\{d_{t+1}\right\}=\left(1+\eta^{-1}\right)^{\beta}\left(\frac{1+\left(1+\eta^{-1}\right)^{1-\alpha-\beta}}{2+\eta^{-1}}\right)
$$

Notice that if the following condition is satisfied which ensures that the global dynamics exhibit multiple steady states,

$$
\frac{\beta}{\alpha} \geq \frac{\ln \left(\frac{2+\eta^{-1}}{1+\left(1+\eta^{-1}\right)^{(1-\alpha-\beta)}}\right)^{\frac{1}{\alpha}}}{\ln \left(1+\eta^{-1}\right)} \equiv \Gamma>\Pi
$$

then $d_{t+1} \geq 1$. Given the evolving pattern of $x_{t}$ in that range of inequality in Region $I I$ where $x_{t} \leq 1+\eta^{-1}$, we also have $d_{t+n} \geq 1$, whatever $n \geq 1$.

\section{References}

[1] Alesina, A.F., and Rodrik, D. (1994) “Distribution Politics and Economic Growth," Quarterly Journal of Economics, 109: $465-490$.

[2] Barham, V., Boadway, R., Marchand, M., and Pestiau, P. (1997) "Volunteer Work and Club Size: Nash Equilibrium and Optimality," Journal of Public Economics, 65: 9-22.

[3] Barro, R.J., and Lee, J. (1993) "International Comparison of Educational Attainment," Journal of Monetary Economics, 32: 363-394.

[4] Bénabou, R. (1996a) "Equity and Efficiency in Human Capital Investment: The Local Connection," Review of Economic Studies, 63: 237-264.

[5] Bénabou, R. (1996b) "Heterogeneity, Stratification, and Growth: Macroeconomic Implications of Community Structure and School Finance," American Economic Review, 86: 584-609. 
[6] Bénabou, R. (1996c) “Inequality and Growth,” in Ben S. Bernanke and Julio J. Rotemberg eds., NBER macro annual, Cambridge, MA: MIT Press, 11: 11-74.

[7] Bénabou, R. (2000) “Unequal Societies: Income Distribution and the Social Contract," American Economic Review, 90: 96-129.

[8] Bolton, P., and Roland, G. (1997) “The Break Up of Nations: A Political Economy Analysis,” Quarterly Journal of Economics, 113: 1057-1090.

[9] Borjas, G.J. (1995) "Ethnicity, Neighborhoods, and Human Capital Externalities," American Economic Review, 85: 365-390.

[10] Breusch, T., and Pagan, , A. (1980). “The LM Test and Its Application to Model Specification in Econometrics,” Review of Economic Studies, 47: 239-254.

[11] Checchi, D., Ichino, A., and Rustichni, A. (1999) "More Equal but Less Mobile? Education Financing and Intergenerational Mobility in Italy and in the U.S.," Journal of Public Economics, 74: 351-393.

[12] Cooper, S.J., Durlauf, S.N., and Johnson, P.A. (1994) “On the Evolution of Economic Status Across Generations," Business and Economics Statistics Section, American Statistical Association: 50-58.

[13] Deininger, K., and Squire, L. (1996) “A New Dataset Measuring Income Inequality,” The World Bank Economic Review, 10: 565-591

[14] Durlauf, S.N. (1994) “Spillovers, Stratification, and Inequality," European Economic Review, 38: 836-845.

[15] Durlauf, S.N. (1996) “A Theory of Persistent Income Inequality,” Journal of Economic Growth, 1: 75-93.

[16] Durlauf, S.N. , and Johnson, P.A. (1995) “Multiple Regimes and Cross-Country Growth Behavior," Journal of Applied Econometrics, 10: 365-384.

[17] Easterly, W., and Rebelo, S. (1993) "Fiscal Policy and Economic Growth,” Journal of Monetary Economics, 32: $417-$ 458.

[18] Epple, D., and Romano, R.E. (1996) "Ends Against the Middle: Determining Public Service Provision When There Are Private Alternatives," Journal of Public Economics, 62: 297-325. 
[19] Farrell, J., and Scotchmer, S. (1988) “Partnerships,” Quarterly Journal of Economics, 103: 279-297.

[20] Fernandez, R., and Rogerson, R. (1995) “On the Political Economy of Education Subsidies,” Review of Economic Studies, 62: 249-262.

[21] Fernandez, R., and Rogerson, R. (1996) “Income Distribution, and the Quality of Education," Quarterly Journal of Economics, : 135-164.

[22] Figini, P. (1999) “Inequality and Growth Revisited,” Trinity Economic Paper Series, N 99/2.

[23] Filmer, D., and Pritchett, L. (2000) “The Effect of Household Wealth on Educational Attainment Around the World: Demographic and Health Survey Evidence," World Bank Working Paper.

[24] Fisher, G.R., and McAleer, M. (1981).“Alternative Procedures and Associated Tests of Significance for Nonnested Hypothesis," Journal of Econometrics, 16: 103-119.

[25] Galland, O., and Rouault, D. (1996) “Des études supérieures inégalement rentables selon les milieux sociaux,” INSEE Première, $N^{\circ} 469$.

[26] Galor, O., and Zeira, J. (1993) “Income Distribution and Macroeconomics,” Review of Economic Studies, 60: 35-52.

[27] Glomm, G., and Ravikumar, B. (1992) “Public Versus Private Investment in Human Capital: Endogenous Growth and Income Inequality,” Journal of Political Economy, 100: 818-834.

[28] Lindert, P.H. (1996) "What Limits Social Spending?” Explorations in Economic History, 33: 1-34.

[29] Llyod-Ellis, H. (2000) "Public Education, Occupational Choice, and the Growth-Inequality Relationship," International Economic Review, 41: 171-201.

[30] Mingat, A., and Tan, J.P. (1985) “On Equity in Education Again: An International Comparison," Journal of Human Resources, 20: 298-308.

[31] Moulin, H. (1983) "The Strategy of Social Choice," North-Holland Publishing Company, eds. C.J. Bliss and M.D. Intriligator.

[32] Perotti, R. (1993) “Political Equilibrium, Income Distribution, and Growth,” Review of Economic Studies, 60: $755-$ 776. 
[33] Perotti, R. (1994) “Income Distribution and Investment,” European Economic Review, 38: 827-835.

[34] Perotti, R. (1996) "Growth, Income Distribution, and Democracy: What the Data Say," Journal of Economic Growth, 1: $149-187$.

[35] Persson, T. and Tabellini, G., (1994) "Is Inequality Harmful for Growth? Theory and Evidence," American Economic Review, 84: 600-621.

[36] Persson, T., and Tabellini, G., (2000) "Political Economics, Explaining Economic Policy," The MIT Press, Cambridge, Massachusetts..

[37] Saint Paul, G. (1994) “The Dynamics of Exclusion and Fiscal Conservatism,” CEPR Discussion Paper No. 998.

[38] Saint Paul, G., and Verdier, T. (1993) "Education, Demography and Growth," Journal of Development Economics, 42: $399-407$.

[39] Solon, G. (1992) "Intergenerational Income Mobility in the United States," American Economic Review, 82: 393-408.

[40] Streufert, P. (2000) "The Effect of Underclass Social Isolation on Schooling Choice," Journal of Public Economic Theory, 2: 461-482.

[41] Tamura, R. (1991) “Income Convergence in an Endogenous Growth Model,” Journal of Political Economy, 99: 522540.

[42] Zimmerman, D.J. (1992) "Regression Toward Mediocrity in Economic Stature," American Economic Review, 82: 408-429. 